\title{
An efficient Poisson solver for complex embedded boundary domains using the multi-grid and fast multipole methods
}

\author{
Narsimha Reddy Rapaka ${ }^{\mathrm{a}}$, Ravi Samtaney ${ }^{\mathrm{a}, *}$ \\ ${ }^{a}$ King Abdullah University of Science and Technology, Thuwal, KSA
}

\section{A R T I C LE INFO}

\section{Article history:}

embedded boundary method, Poisson solver, geometric multigrid method, Green's function, boundary element method (BEM), fast multipole method (FMM)

\begin{abstract}
A B S T R A C T
We present an efficient method to solve the Poisson equation in embedded boundary (EB) domains. The original problem is divided into an inhomogeneous problem without the effects of $\mathrm{EB}$ and a homogeneous problem that imposes the effects of EB. The inhomogeneous problem is efficiently solved through a geometric multi-grid (GMG) solver and the homogenous problem is solved through a boundary element method (BEM) utilizing the free space Green's function. Our method is robust and can handle sharp geometric features without any special treatment. Analytical expressions are presented for the boundary and the domain integrals in BEM to reduce the computational cost and integration error relative to numerical quadratures. Furthermore, a fast multipole method (FMM) is employed to evaluate the boundary integrals in BEM and reduce the computational complexity of BEM. Our method inherits the complementary advantages of both GMG and FMM and presents an efficient alternative with linear computational complexity even for problems involving complex geometries. We observe that the overall computational cost is an order of magnitude lower compared with a stand-alone FMM and is similar to that of an ideal GMG solver.
\end{abstract}

(c) 2020 Elsevier Inc. All rights reserved.

\footnotetext{
${ }^{*}$ Corresponding author. Tel.: +966 12 8082958. The code used in this study is available upon request addressed to the corresponding author. e-mail: Ravi.Samtaney@kaust.edu.sa (Ravi Samtaney)
} 


\section{Introduction}

Solution of the Poisson equation (an elliptic equation) is important in a variety of engineering and physics applications. Of particular interest is the numerical solution of incompressible Navier-Stokes equations by projection methods wherein the pressure is obtained by solving a Poisson equation. A variety of solution methodologies exist such as FFT, multigrid methods, boundary integral methods such as fast multipole method (FMM), Krylov subspace methods such as GMRES, BiCGSTAB, etc. The particular choice is largely influenced by computational efficiency and scope with regard to geometry, mesh, boundary conditions and type of PDE. The present work focuses on constant coefficient Poisson equation with emphasis on complex embedded boundary (EB) domains and arbitrary boundary conditions (Dirichlet, Neumann, Mixed and Periodic).

While FFT based methods are the most efficient for smooth source functions (Gholami et al., 2016), their applicability is generally limited for problems with infinite/periodic domains and uniform Cartesian grids. For non-periodic domains, geometric multigrid methods (GMG) are the most efficient, however, they suffer from poor convergence for problems with anisotropic grids and complex geometries. Although performance of GMG for anisotropic grids may be improved significantly through semi-coarsening algorithms (Larsson et al., 2005), handling complex geometry with sharp features is still a major challenge faced by GMG. In particular, the multi-level representation of complex geometry features is complicated and ambiguous because small scale geometrical features are lost or poorly represented on coarse grid levels leading to a worsening impact on the convergence behavior. Operators at coarser grid levels are not faithful approximations to the operator at the finest grid which strongly affects the performance. Sometimes, deletion of some of the coarsest levels from, say the V-cycle, may improve convergence, however, this depends on the geometrical features, and may lead to loss of linear computational complexity (Guillet and Teyssier, 2011; Crockett et al., 2011; Coco and Russo, 2013, 2018). Some works modify restriction/prolongation operators through volume fractions of the interior/exterior to account for small scale geometrical features at coarser levels. This method is complicated, expensive (particularly, for moving boundaries) and still results in loss of linear complexity. Hosseinverdi and Fasel (2018), Coco and Russo (2013, 2018) modified restriction/prolongation stencils near the EB and the latter found improved convergence by additional smoothing sweeps along the boundary.

Crockett et al. (2011) developed a Poisson solver with discontinuous coefficients across the EB based on the cut-cell approach wherein fluxes over cells cut by the EB are treated through a finite volume method. However, convergence (number of MG iterations) of their method depends on the number of unknowns, and the dependence is stronger for larger difference in coefficients across the EB. Devendran et al. (2017) developed a fourth order EB Poisson solver based on the cut-cell approach and demonstrated convergence for sharp complex geometries $\left(C^{0}\right)$ through geometric regularization. Algebraic multigrid method is a popular alternative for complex geometries, however, it suffers from significant memory/storage requirements.

Alternatively, the boundary element method (BEM) is one of the most efficient methods to solve elliptic problems involving complex geometries due to the fact that the discrete degrees of freedom are distributed on a one dimensional representation of the complex boundary contour embedded in a 2D domain. Usually, inhomogeneous problems involve $O\left(N^{2}\right)$ operations for problems with $N$ degrees of freedom. However, specialized methods such as fast multipole method (FMM) brings the operation count down to $O(N)$ by trading (tunable) accuracy. Originally, Carrier et al. (1988); Cheng et al. (1999) developed FMM to accelerate N-body particle computations. Later, Ethridge and Greengard (2001); Langston et al. (2011) presented modifications to the particle-FMM to obtain a volume FMM wherein summation over source points is replaced by an integral over the continuous source density.

Liska and Colonius (2014) developed a fast Poisson solver using lattice Green's functions (discrete analogue of Green's function on a Cartesian grid) by leveraging the efficiency of FFT on Cartesian grids. However, their method is limited to unbounded domains with a uniform Cartesian mesh. They extended the lattice Green's function approach to solve external incompressible viscous flows on unbounded domains (Liska and Colonius, 2016) and over immersed boundaries (Liska and Colonius, 2017). Kavouklis and Colella (2019) employed method of local corrections to solve Poisson equation on infinite domains/unit cube with periodic boundary conditions. Askham and Cerfon (2017) developed a Poisson solver for complex geometries using the fast multipole method (FMM). They split the original problem into homogeneous and inhomogeneous problems. Notably, they employed a fast direct solver instead of using iterative solvers.

For homogeneous elliptic problems such as the Laplace equation, the boundary integral approach (e.g., BEM) stands out to be the most efficient method for complex geometries with non-periodic boundary conditions. However, Green's function based approaches are generally limited to constant coefficient problems. Although both GMG and FMM deliver linear computational complexity for the Poisson equation (Eq. (1)) in Cartesian geometries, FMM has 
a much larger constant in the complexity estimates compared with GMG (Gholami et al., 2016). We observed in our numerical tests (Sec. 5) that $t_{F M M}^{i}>>t_{F M M}^{h} \sim t_{G M G}$, where $t_{G M G}$ and $t_{F M M}^{i}$ are the costs of ideal (Cartesian domain) GMG and FMM, respectively, for inhomogeneous problems and $t_{F M M}^{h}$ is the cost of FMM for homogeneous problems $(S=0$ in Eq. (1)).

Our method divides the original problem into two parts similar to Askham and Cerfon (2017): an inhomogeneous problem without the effects of EB, and a homogeneous problem that imposes the effects of EB. In contrast to Askham and Cerfon (2017), the inhomogeneous problem is solved efficiently through GMG in $O(N)$ operations instead of FMM. The homogeneous problem is solved through BEM/FMM and includes mixed boundary conditions (both Dirichlet and Neumann) while Askham and Cerfon (2017) formulated the homogeneous problem via layer potentials limited to Dirichlet boundary conditions. The present approach provides a simple, efficient and versatile alternative to the existing approaches (Coco and Russo, 2013, 2018) and eliminates the need for multi-level representation of EB. The present approach is novel in that it integrates GMG and FMM and outperforms stand-alone FMM (Askham and Cerfon, 2017) by taking advantage of the higher efficiency of GMG over FMM for inhomogeneous problems (demonstrated in Sec. 5), utilizes analytical integration for both boundary and domain integrals, and efficient adaptive quadtrees optimized separately for boundary and domain integrals. Remarkably, the combined GMG-FMM solver has linear complexity for solving Poisson equation in complex geometries with computational cost similar to that of an ideal GMG.

Numerical quadratures are widely used to compute the boundary integrals in BEM. Typically, a high degree of accuracy is essential to compute the layer potentials involving weakly singular integrands which increases the computational cost. In the present work, analytical integration is used to evaluate all the boundary integrals to avoid additional computational expense and errors associated with numerical quadratures. Moreover, in our present method, no special treatment is required when the EB includes sharp features.

To demonstrate the effect of the presence of EB on convergence of GMG, a simple alternative is considered in the present work in which the EB condition is applied only at the finest grid level in the GMG solver. The EB is excluded from coarser grid levels (restriction/prolongation operations) and hence coarser grids do not sense the presence of EB. While this is a simple idea that is relatively easily implemented, we note that the computational cost increases and perhaps outweighs the simplicity of this approach.

A brief outline of the paper is as follows. The problem formulation is described in Sec. 2. In Sec. 3, the boundary element method is described. FMM and an adaptive quadtree data structure for two dimensional homogeneous problems are briefly discussed in Sec. 4. Results including accuracy and performance of the present method are presented for a variety of complex geometry cases in Sec. 5 and the combined GMG-FMM method is demonstrated to be faster by an order of magnitude compared with a stand-alone FMM. A stand-alone FMM used as a performance benchmark in this study is described in Appendix B.

\section{Problem formulation}

Let us denote a Cartesian domain with $\Omega_{c}$ and its boundary with $\Gamma_{c}$. Suppose that $\Omega_{c}$ contains a closed embedded boundary $\Gamma_{e b}$ partitioning $\Omega_{c}$ into $\Omega_{c}^{i}$ (interior of $\Gamma_{e b}$ ) and $\Omega_{c}^{e}$ (exterior of $\Gamma_{e b}$ ). Let $\Omega$ be the domain of interest and $\Gamma$ be its boundary. For exterior problems, $\Omega=\Omega_{c}^{e}$ with $\Gamma=\Gamma_{c} \cup \Gamma_{e b}$ and for interior problems, $\Omega=\Omega_{c}^{i}$ with $\Gamma=\Gamma_{e b}$. Let $\psi$ be a signed distance function which is negative inside $\Omega$, positive outside $\Omega$ and zero on $\Gamma_{e b}$. We aim to solve the Poisson equation with source $S(\boldsymbol{x})$ in $\Omega$ and Dirichlet/Neumann/Mixed boundary conditions on $\Gamma$.

$$
\begin{aligned}
\nabla^{2} p & =S(\boldsymbol{x}) \quad \text { in } \Omega \\
a p+b \frac{\partial p}{\partial n} & =f(\boldsymbol{x}) \quad \text { on } \Gamma_{c}, \quad c p+d \frac{\partial p}{\partial n}=g(\boldsymbol{x}) \quad \text { on } \Gamma_{e b}
\end{aligned}
$$

where, $\frac{\partial p}{\partial n}=\nabla p \cdot \hat{\boldsymbol{n}}$ and $\hat{\boldsymbol{n}}$ is the unit boundary normal pointing into $\Omega$. The coefficients $a, b$ and $c, d$ can be arbitrary and their convex combination yield Dirichlet boundary conditions for $b=0, d=0$ and Neumann boundary conditions for $a=0, c=0$. We apply the principle of linear superposition to split the solution $p$ into inhomogeneous $\left(p^{i}\right)$ and homogeneous $\left(p^{h}\right)$ parts so that $p=p^{i}+p^{h}$ and solve the following equations,

$$
\begin{aligned}
& \nabla^{2} p^{i}=S(\boldsymbol{x}) \text { in } \Omega_{c}, \quad a p^{i}+b \frac{\partial p^{i}}{\partial n}=f(\boldsymbol{x}) \text { on } \Gamma_{c}, \\
& \nabla^{2} p^{h}=0 \quad \text { in } \Omega, \quad a p^{h}+b \frac{\partial p^{h}}{\partial n}=0 \quad \text { on } \Gamma_{c}, \quad \text { and } c p^{h}+d \frac{\partial p^{h}}{\partial n}=\tilde{g}(\boldsymbol{x}) \text { on } \Gamma_{e b} .
\end{aligned}
$$


where, $\tilde{g}(\boldsymbol{x})=g(\boldsymbol{x})-\left(c p^{i}+d \frac{\partial p^{i}}{\partial n}\right)$. The inhomogeneous problem is discretized with a standard second order central finite difference scheme and solved through a standard V-cycle GMG with two Red-Black point Gauss-Siedel sweeps during each pre and post smoothing operations. The homogeneous problem is solved via BEM described in Sec. 3. Since conventional BEM has $O\left(N^{3 / 2}\right)$ complexity for 2D homogeneous problems of size $N$, a fast multipole method (FMM) described in Sec. 4 is employed to achieve linear complexity. Both GMG and FMM are implemented in MATLAB R2017b and a bulit-in GMRES solver is used to solve dense linear systems encountered in BEM/FMM. Boundary conditions for $p^{h}$ on $\Gamma_{e b}$ in Eq. (4) require evaluation of $p^{i}, \frac{\partial p^{i}}{\partial n}$ (thereby $\tilde{g}(\boldsymbol{x})$ ) at nodes on $\Gamma_{e b}$ from $p^{i}$ available at Cartesian grid nodes which is described in the following sections.

\subsection{Bilinear reconstruction}

Let a node $\boldsymbol{x}_{s}=\left(x_{s}, y_{s}\right)$ on $\Gamma_{e b}$ with unit normal $\hat{\boldsymbol{n}}=\left(n_{x}, n_{y}\right)$ be surrounded by four Cartesian nodes $P_{1}, P_{2}, P_{3}, P_{4}$ with indices $(i, j),(i+1, j),(i, j+1),(i+1, j+1)$, respectively. Define $\Delta x=x_{i+1}-x_{i}, \Delta y=y_{j+1}-y_{j}, \Delta x_{1}=$ $x_{s}-x_{i}, \Delta x_{2}=x_{i+1}-x_{s}$, and $\Delta y_{1}=y_{s}-y_{j}, \Delta y_{2}=y_{j+1}-y_{s}$. A bilinear approximation at $\boldsymbol{x}_{s}$ involves four degrees of freedom determined from field values at $P_{1}, P_{2}, P_{3}, P_{4}$ and yields following expressions,

$$
\begin{aligned}
p\left(\boldsymbol{x}_{s}\right) & =\frac{\Delta y_{1}\left[\Delta x_{1} p_{i+1, j+1}+\Delta x_{2} p_{i, j+1}\right]+\Delta y_{2}\left[\Delta x_{1} p_{i+1, j}+\Delta x_{2} p_{i, j}\right]}{\Delta x \Delta y}, \\
\frac{\partial p}{\partial n}\left(\boldsymbol{x}_{s}\right) & =n_{x}\left(\frac{\Delta y_{2}\left(p_{i+1, j}-p_{i, j}\right)+\Delta y_{1}\left(p_{i+1, j+1}-p_{i, j+1}\right)}{\Delta x \Delta y}\right)+n_{y}\left(\frac{\Delta x_{2}\left(p_{i, j+1}-p_{i, j}\right)+\Delta x_{1}\left(p_{i+1, j+1}-p_{i+1, j}\right)}{\Delta x \Delta y}\right) .
\end{aligned}
$$

\subsection{Bicubic reconstruction}

In this case, let $\boldsymbol{x}_{s}$ be surrounded by 16 neighboring Cartesian nodes $\left(x_{m}, y_{n}\right), m, n=1,2,3,4$, denoted by $P_{m, n}$. Define $\tilde{x}=x-x_{s}, \tilde{y}=y-y_{s}$ and the bicubic polynomial $p(\tilde{x}, \tilde{y})=\sum_{m=0}^{3} \sum_{n=0}^{3} C_{m n} \tilde{x}^{m} \tilde{y}^{n}$. A bicubic approximation at $\boldsymbol{x}_{s}$, with a formal accuracy of $O\left(\Delta x^{4}, \Delta y^{4}\right)$, involves sixteen degrees of freedom which are determined from field values at $P_{m, n}=\left(x_{m}, y_{n}\right)$. The solution and its normal derivative at $\boldsymbol{x}_{s}$ are given by,

$$
\begin{aligned}
p\left(x_{s}, y_{s}\right) & =\sum_{n=1}^{4} v_{n}(\tilde{y}) \sum_{m=1}^{4} v_{m}(\tilde{x}) p\left(x_{m}, y_{n}\right), \quad \frac{\partial p}{\partial n}\left(\boldsymbol{x}_{s}\right)=n_{x} \frac{\partial p}{\partial x}\left(\boldsymbol{x}_{s}\right)+n_{y} \frac{\partial p}{\partial y}\left(\boldsymbol{x}_{s}\right), \\
\frac{\partial p}{\partial x}\left(x_{s}, y_{s}\right) & =\sum_{n=1}^{4} v_{n}(\tilde{y}) \sum_{m=1}^{4} w_{m}(\tilde{x}) p\left(x_{m}, y_{n}\right), \quad \frac{\partial p}{\partial y}\left(x_{s}, y_{s}\right)=\sum_{m=1}^{4} v_{m}(\tilde{x}) \sum_{n=1}^{4} w_{n}(\tilde{y}) p\left(x_{m}, y_{n}\right) .
\end{aligned}
$$

where, the weights $v_{m}(\tilde{x})$ and $w_{m}(\tilde{x})$ depend on $\tilde{x}_{m}$ as below (the weights $v_{n}(\tilde{y})$ and $w_{n}(\tilde{y})$ depend on $\tilde{y}_{n}$ similarly),

$$
\begin{aligned}
& v_{1}(\tilde{x})=\frac{\tilde{x}_{2} \tilde{x}_{3} \tilde{x}_{4}}{f\left(\tilde{x}_{1} ; \tilde{x}_{2}, \tilde{x}_{3}, \tilde{x}_{4}\right)}, \quad v_{2}(\tilde{x})=\frac{\tilde{x}_{1} \tilde{x}_{3} \tilde{x}_{4}}{f\left(\tilde{x}_{2} ; \tilde{x}_{1}, \tilde{x}_{3}, \tilde{x}_{4}\right)}, \quad v_{3}(\tilde{x})=\frac{\tilde{x}_{1} \tilde{x}_{2} \tilde{x}_{4}}{f\left(\tilde{x}_{3} ; \tilde{x}_{1}, \tilde{x}_{2}, \tilde{x}_{4}\right)}, \quad v_{4}(\tilde{x})=\frac{\tilde{x}_{1} \tilde{x}_{2} \tilde{x}_{3}}{f\left(\tilde{x}_{4} ; \tilde{x}_{1}, \tilde{x}_{2}, \tilde{x}_{3}\right)}, \\
& w_{1}(\tilde{x})=\frac{-\left(\tilde{x}_{2} \tilde{x}_{3}+\tilde{x}_{2} \tilde{x}_{4}+\tilde{x}_{3} \tilde{x}_{4}\right)}{f\left(\tilde{x}_{1} ; \tilde{x}_{2}, \tilde{x}_{3}, \tilde{x}_{4}\right)}, \quad w_{2}(\tilde{x})=\frac{-\left(\tilde{x}_{1} \tilde{x}_{3}+\tilde{x}_{1} \tilde{x}_{4}+\tilde{x}_{3} \tilde{x}_{4}\right)}{f\left(\tilde{x}_{2} ; \tilde{x}_{1}, \tilde{x}_{3}, \tilde{x}_{4}\right)}, \quad w_{3}(\tilde{x})=\frac{-\left(\tilde{x}_{1} \tilde{x}_{2}+\tilde{x}_{1} \tilde{x}_{4}+\tilde{x}_{2} \tilde{x}_{4}\right)}{f\left(\tilde{x}_{3} ; \tilde{x}_{1}, \tilde{x}_{2}, \tilde{x}_{4}\right)}, \\
& w_{4}(\tilde{x})=\frac{-\left(\tilde{x}_{1} \tilde{x}_{2}+x_{1} x_{3}+x_{2} x_{3}\right)}{f\left(x_{4} ; x_{1}, x_{2}, x_{3}\right)}, \quad f(a ; b, c, d)=b c d-a(b c+b d+c d)+a^{2}(b+c+d)-a^{3} .
\end{aligned}
$$

\section{Boundary element method (BEM)}

Here, we describe the boundary element method (Pozrikidis, 2002, 2008) used to solve the homogeneous problem (Eq. (4)). For brevity, we omit the superscript $h$ used for the homogeneous solution in this section. An extension to the inhomogeneous problem (Eq. 1) is described in Appendix B.3. 


\subsection{Integral representation}

Let $G\left(x, x_{0}\right)$ be the fundamental solution of the Laplace operator satisfying,

$$
\nabla^{2} G\left(x, x_{0}\right)+\delta\left(x-x_{0}\right)=0,
$$

where $\delta$ is the Dirac delta distribution. Apply Green's second identity to the solution $p$ and the Green's function $G$ to have,

$$
G\left(\boldsymbol{x}, \boldsymbol{x}_{0}\right) \nabla^{2} p(\boldsymbol{x})-p(\boldsymbol{x}) \nabla^{2} G\left(\boldsymbol{x}, \boldsymbol{x}_{0}\right)=\nabla \cdot\left[G\left(\boldsymbol{x}, \boldsymbol{x}_{0}\right) \nabla p(\boldsymbol{x})-p(\boldsymbol{x}) \nabla G\left(\boldsymbol{x}, \boldsymbol{x}_{0}\right)\right] .
$$

Substitute Eq. (4),(9) in Eq. (10), integrate over $\Omega$ and apply divergence theorem to have the Green's third identity,

$$
\int_{\Omega} p(\boldsymbol{x}) \delta\left(\boldsymbol{x}-\boldsymbol{x}_{0}\right) d \Omega=-\int_{\Gamma} G\left(\boldsymbol{x}, \boldsymbol{x}_{0}\right) \nabla p(\boldsymbol{x}) \cdot \hat{\boldsymbol{n}} d \Gamma+\int_{\Gamma}^{P V} p(\boldsymbol{x}) \nabla G\left(\boldsymbol{x}, \boldsymbol{x}_{0}\right) \cdot \hat{\boldsymbol{n}} d \Gamma .
$$

where, $\hat{\boldsymbol{n}}$ is the unit inward normal vector on $\Gamma$. Note that the last integral in the above equation is a principal value integral when $x_{0}$ lies on $\Gamma$ because $\nabla G\left(x, x_{0}\right)$ is singular. Using the properties of the Dirac delta distribution, the integral on the left side of Eq. (11) becomes,

$$
\int_{\Omega} p(\boldsymbol{x}) \delta\left(\boldsymbol{x}-\boldsymbol{x}_{0}\right) d \Omega=\alpha p\left(\boldsymbol{x}_{0}\right)
$$

where, $\alpha$ is equal to 1 for $\boldsymbol{x}_{0} \in \Omega, 0$ for $\boldsymbol{x}_{0} \notin \Omega, 1 / 2$ for $\boldsymbol{x}_{0}$ on smooth part of $\Gamma$, and $\theta /(2 \pi)$ for $\boldsymbol{x}_{0}$ on sharp corner of $\Gamma$ with $\theta$ being the angle subtended by $\Gamma$ at the corner $\boldsymbol{x}_{0}$ towards $\Omega(\$ 2.4 .1$, Pozrikidis (2002)). For example, corners of a square domain yield $\alpha=1 / 4$ with $\theta=\pi / 2$. Using Eq. (11) and (12), we obtain the following integral representations for $p(\boldsymbol{x})$ on the boundary $\Gamma$ (assuming that $\boldsymbol{x}_{0}$ lies only on smooth part of $\Gamma$ ) and in the domain $\Omega$,

$$
\begin{array}{rlrl}
\frac{1}{2} p\left(\boldsymbol{x}_{0}\right) & =-\int_{\Gamma} G\left(\boldsymbol{x}, \boldsymbol{x}_{0}\right) \frac{\partial p}{\partial n}(\boldsymbol{x}) d \Gamma+\int_{\Gamma}^{P V} p(\boldsymbol{x}) \frac{\partial G}{\partial n}\left(\boldsymbol{x}, \boldsymbol{x}_{0}\right) d \Gamma, & \boldsymbol{x}_{0} \in \Gamma \\
p\left(\boldsymbol{x}_{0}\right) & =-\int_{\Gamma} G\left(\boldsymbol{x}, \boldsymbol{x}_{0}\right) \frac{\partial p}{\partial n}(\boldsymbol{x}) d \Gamma+\int_{\Gamma} p(\boldsymbol{x}) \frac{\partial G\left(\boldsymbol{x}, \boldsymbol{x}_{0}\right)}{\partial n} d \Gamma, \quad \boldsymbol{x}_{0} \in \Omega
\end{array}
$$

Typically, the boundary conditions provide either $p$ or $\frac{\partial p}{\partial n}$ on different segments of the boundary $\Gamma$ leading to a mix of Fredholm integral equations of first kind and second kind. So, we first solve Eq. (13) for the unknowns (either $p$ or $\frac{\partial p}{\partial n}$ ) on all the boundary segments. Once both $p$ and $\frac{\partial p}{\partial n}$ are known on the entire boundary $\Gamma$, we obtain $p$ inside the domain $\Omega$ through direct evaluation of Eq. (14).

\subsection{BEM discretization}

We discretize the boundary $\Gamma$ into $M$ boundary elements, and the unknown functions $p$ and $q \equiv \frac{\partial p}{\partial n}$ are approximated with constant functions over each element $\Gamma_{i}$, denoted by $p_{i}$ and $q_{i}$, respectively. Henceforth, this approximation is referred as constant element approximation. Applying Eq. (13) at $M$ collocation points (center of each boundary element $\Gamma_{j}$ ) results in a system of dense linear equations (Eq. (15)) for the unknown element value, $p_{i}$ or $q_{i}$. Similarly, applying Eq. (14) at $N$ collocation points corresponding to the interior nodes in $\Omega$ results in $N$ algebraic equations (Eq. (16)). The integral equations Eq. (13), (14) are then approximated by the following algebraic equations,

$$
\begin{aligned}
\frac{1}{2} p\left(\boldsymbol{x}_{j}\right) & =-\sum_{i=1}^{M} q_{i} A_{i}\left(\boldsymbol{x}_{j}\right)+\sum_{i=1}^{M} p_{i} B_{i}\left(\boldsymbol{x}_{j}\right), & \boldsymbol{x}_{j} \in \Gamma, & j=1,2, \ldots, M, \\
p\left(\boldsymbol{x}_{j}\right) & =-\sum_{i=1}^{M} q_{i} A_{i}\left(\boldsymbol{x}_{j}\right)+\sum_{i=1}^{M} p_{i} B_{i}\left(\boldsymbol{x}_{j}\right), & \boldsymbol{x}_{j} \in \Omega, & j=1,2, \ldots, N,
\end{aligned}
$$

where, $A_{i}\left(\boldsymbol{x}_{j}\right) \equiv \int_{\Gamma_{i}} G\left(\boldsymbol{x}, \boldsymbol{x}_{j}\right) d \Gamma$ and $B_{i}\left(\boldsymbol{x}_{j}\right) \equiv \int_{\Gamma_{i}}^{P V} \frac{\partial G\left(\boldsymbol{x}, \boldsymbol{x}_{j}\right)}{\partial n} d \Gamma$ are influence coefficients computed over an individual element $i$. First, Eq. (15) is solved through either direct solvers (Gauss elimination, LU based, etc.) or iterative solvers such as GMRES. For the examples in Sec. 5, we use GMRES as it was faster than LU based solvers for the chosen examples. Then, Eq. (16) is evaluated at $N$ interior nodes in $\Omega$. 
Note that the Green's function property requires $\sum_{i=1}^{M} B_{i}\left(\boldsymbol{x}_{j}\right)=\frac{1}{2}$ for $\boldsymbol{x}_{j}$ on $\Gamma$. In two dimensions, the free-space Green's function is well known to be $G\left(x, x_{0}\right)=-\frac{1}{2 \pi} \ln \left|x-x_{0}\right|$, and

$$
A_{j i} \equiv A_{i}\left(\boldsymbol{x}_{j}\right) \equiv-\frac{1}{2 \pi} \int_{\Gamma_{i}} \ln \left|\boldsymbol{x}-\boldsymbol{x}_{j}\right| d \Gamma, \quad B_{j i} \equiv B_{i}\left(\boldsymbol{x}_{j}\right) \equiv-\frac{1}{2 \pi} \int_{\Gamma_{i}}^{P V} \frac{\hat{\boldsymbol{n}}(\boldsymbol{x}) \cdot\left(\boldsymbol{x}-\boldsymbol{x}_{j}\right)}{\left|\boldsymbol{x}-\boldsymbol{x}_{j}\right|^{2}} d \Gamma,
$$

where $P V$ denotes the Cauchy principal value. Computation of $A_{j i}$ and $B_{j i}$ for linear elements is described below. Curved boundary segments are linearized by partitioning in to a number of straight/linear elements. Let $\Delta l_{i}$ be the length of an element $\Gamma_{i}$ for $2 \mathrm{D}$ problems.

\subsubsection{Singular case $(i=j)$}

For a straight element $\Gamma_{i}$ with $\boldsymbol{x}, \boldsymbol{x}_{i} \in \Gamma_{i}, \hat{\boldsymbol{n}}(\boldsymbol{x}) \cdot\left(\boldsymbol{x}-\boldsymbol{x}_{i}\right)=0$ and the singular case $(i=j)$ yields (Pozrikidis, 2008),

$$
\begin{aligned}
A_{i i} & \equiv A_{i}\left(\boldsymbol{x}_{i}\right) \equiv-\frac{1}{2 \pi} \int_{\Gamma_{i}} \ln \left|\boldsymbol{x}-\boldsymbol{x}_{i}\right| \mathrm{d} l(\boldsymbol{x})=-\frac{1}{\pi} \int_{0}^{\Delta l_{i} / 2} \ln l^{\prime} d l^{\prime}=-\frac{1}{\pi} \frac{\Delta l_{i}}{2}\left(\ln \frac{\Delta l_{i}}{2}-1\right), \\
B_{i i} & \equiv B_{i}\left(\boldsymbol{x}_{i}\right) \equiv-\frac{1}{2 \pi} \int_{\Gamma_{i}}^{P V} \frac{\hat{\boldsymbol{n}}(\boldsymbol{x}) \cdot\left(\boldsymbol{x}-\boldsymbol{x}_{i}\right)}{\left|\boldsymbol{x}-\boldsymbol{x}_{i}\right|^{2}} \mathrm{~d} l(\boldsymbol{x})=0 .
\end{aligned}
$$

\subsubsection{Non-singular case $(i \neq j)$}

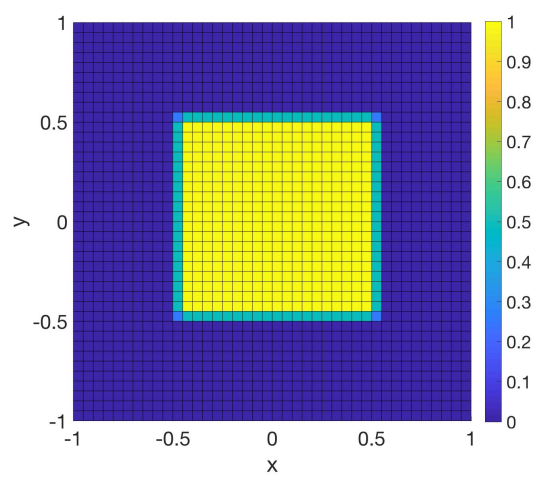

(a)

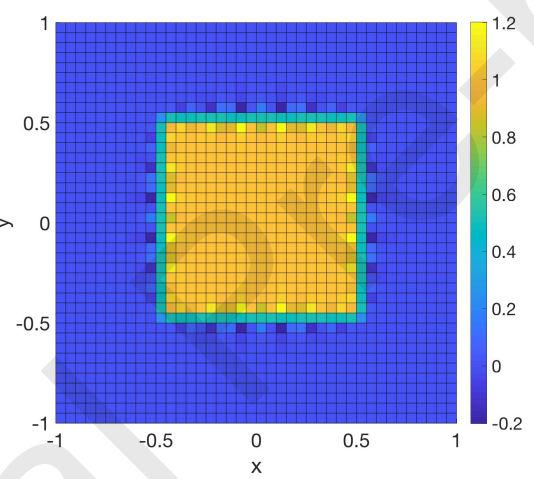

(b)

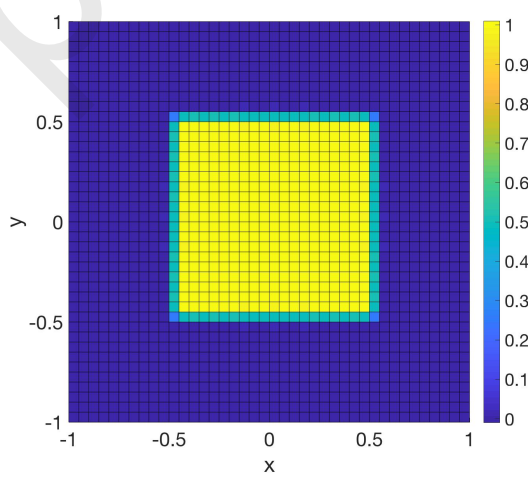

(c)

Fig. 1: Double layer potential $\sum_{i} B_{j i}$ for $\Gamma$, which is a square in this illustration $(x, y \in[-0.5,0.5])$, computed through (a) analytic integration Eq (21), and Gauss-Legendre quadrature with (b) 8 and (c) 20 base points. As expected, $\sum_{i} B_{i}\left(\boldsymbol{x}_{j}\right)=\sum_{i}-\frac{\theta_{j i}}{2 \pi}=\int_{\Gamma}^{P V} \frac{\partial G\left(\boldsymbol{x}, \boldsymbol{x}_{j}\right)}{\partial n} d \Gamma=\alpha=1,0,0.5,0.25$ for $\boldsymbol{x}_{j}$ located on the inside, the outside, the edges and the corners of the square, respectively.

Conventionally, numerical quadratures are used to compute the influence coefficients $\left(A_{j i}, B_{j i}\right)$. Since the integrands are weakly singular, high accuracy is required (see Fig. 1 for an example demonstrating this). Here, we present analytical expressions for $A_{j i}, B_{j i}$ in case of straight boundary elements so that the computation is efficient and has no error due to numerical integration. Note that the analytical expressions are applicable for even the singular case $(i=j)$.

Let $\hat{\boldsymbol{t}}=\left(\boldsymbol{x}_{i+1}-\boldsymbol{x}_{i}\right) /\left|\boldsymbol{x}_{i+1}-\boldsymbol{x}_{i}\right|$ be the unit vector along an element $\Gamma_{i}$, and $l$ be the distance from the element midpoint $\boldsymbol{x}_{m}$ along $\hat{\boldsymbol{t}}$, see Fig. 2(a,b). For straight elements with $\boldsymbol{x} \in\left[\boldsymbol{x}_{i}, \boldsymbol{x}_{i+1}\right]$, define $r(\boldsymbol{x}) \equiv\left|\boldsymbol{x}-\boldsymbol{x}_{j}\right|, r_{m} \equiv r\left(\boldsymbol{x}_{m}\right)$. Now, let us express $\boldsymbol{x}_{m}-\boldsymbol{x}_{j}$ in terms of an orthonormal basis $\hat{\boldsymbol{n}}, \hat{\boldsymbol{t}}$ (see Fig. 2 (a,b)) with components $a, b$, respectively, where $a=\left(\boldsymbol{x}_{m}-\boldsymbol{x}_{j}\right) \cdot \hat{\boldsymbol{n}}$ and $b=\left(\boldsymbol{x}_{m}-\boldsymbol{x}_{j}\right) \cdot \hat{\boldsymbol{t}}$. Then, we have $\boldsymbol{x}-\boldsymbol{x}_{j}=a \hat{\boldsymbol{n}}+(b+l) \hat{\boldsymbol{t}}$ and $r^{2}=a^{2}+(b+l)^{2}$. Define $c \equiv b+\Delta l_{i} / 2, d \equiv b-\Delta l_{i} / 2$. Note that the integrand below is weakly singular whose integral is a continuous function.

$$
\begin{aligned}
A_{j i} & \equiv A_{i}\left(\boldsymbol{x}_{j}\right) \equiv-\frac{1}{2 \pi} \int_{-\Delta l_{i} / 2}^{\Delta l_{i} / 2} \ln \left|\boldsymbol{x}-\boldsymbol{x}_{j}\right| \mathrm{d} l(\boldsymbol{x})=-\frac{1}{2 \pi}\left[c \ln \left|a^{2}+c^{2}\right|^{1 / 2}-d \ln \left|a^{2}+d^{2}\right|^{1 / 2}-\Delta l_{i}+a \theta_{j i}\right], \\
B_{j i} & \equiv B_{i}\left(\boldsymbol{x}_{j}\right) \equiv-\frac{1}{2 \pi} \int_{-\Delta l_{i} / 2}^{\Delta l_{i} / 2} \frac{\hat{\boldsymbol{n}}(\boldsymbol{x}) \cdot\left(\boldsymbol{x}-\boldsymbol{x}_{j}\right)}{\left|\boldsymbol{x}-\boldsymbol{x}_{j}\right|^{2}} \mathrm{~d} l(\boldsymbol{x})=-\frac{\theta_{j i}}{2 \pi}, \quad \theta_{j i}=\tan ^{-1}\left(\frac{a \Delta l_{i}}{a^{2}+c d}\right) .
\end{aligned}
$$




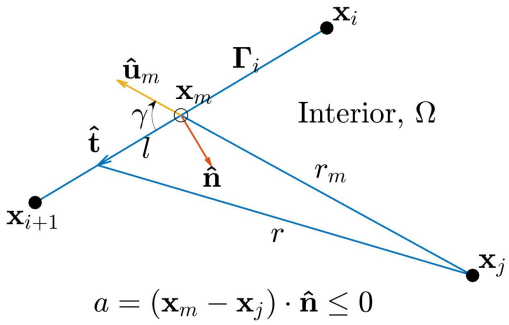

(a)

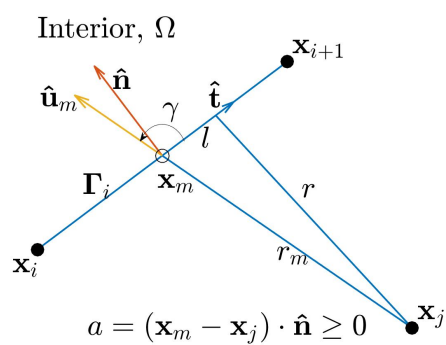

(b)

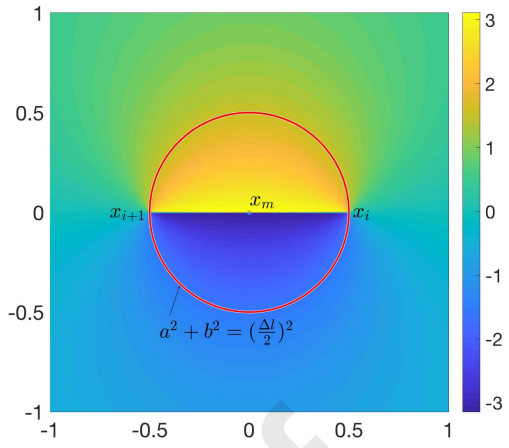

(c)

Fig. 2: Schematic of a straight element $\Gamma_{i} \in\left[\boldsymbol{x}_{i}, \boldsymbol{x}_{i+1}\right]$ with source at $\boldsymbol{x}_{j}$. The boundary element $\Gamma_{i}$ is in (a) anti-clockwise $\gamma \in[-\pi, 0](a \leq 0)$, (b) clockwise $\gamma \in[0, \pi](a \geq 0)$ orientation w.r.t. $x_{0}$. The unit inward normal $\hat{\boldsymbol{n}}$ always points in to the interior $\Omega$. (c) Distribution of $\theta_{j i}$, angle subtended by the element $\Gamma_{i}$ at the source $\boldsymbol{x}_{j}$, with $\Delta l=1, \hat{\boldsymbol{t}}=(-1,0)$, and $\hat{\boldsymbol{n}}=(0,-1)$.

Proofs of Eq. (20) and (21) are given in Appendix A. Here, $\theta_{j i} \in[-\pi, \pi]$ is the angle subtended by each boundary element $\Gamma_{i}=\left[\boldsymbol{x}_{i}, \boldsymbol{x}_{i+1}\right]$ at the source $\boldsymbol{x}_{j}$ with $\hat{\boldsymbol{t}}$ counter-clockwise being positive and clockwise being negative. Note that $\sum_{i} \theta_{j i}=2 \pi, 0, \pi$ for $\boldsymbol{x}_{j}$ on the interior, the exterior and the boundary of the closed surface. If $\boldsymbol{x}_{j}$ is located on the sharp corner of a non-smooth boundary, $\theta_{j i}$ is the subtended angle at the sharp corner towards the interior (Fig. 1).

Since $\theta_{j i}$ is discontinuous as $\boldsymbol{x}_{j}$ approaches the element $\Gamma_{i}$ (see Fig. 2c), we explicitly assign $\theta_{j i}=0$ for $|a| \leq 10^{-15}$, in accordance with the singular case Eq. (19), to avoid $\theta_{j i}= \pm \pi$ due to round-off errors in $\boldsymbol{x}_{i}, \boldsymbol{x}_{i+1}, \boldsymbol{x}_{j}$.

\subsubsection{Effect of linearization of $E B$}

For circular and other non-linear boundaries, we linearize the EB by dividing it into a number of linear segments. This creates non-physical sharp corners at the ends of each boundary element and may lead to errors in $\sum_{i} \theta_{j i}$ if $\boldsymbol{x}_{j}$ coincides with the element end points and thereby the non-physical corners. However, with increasing resolution, $\theta_{j i}$ converges to its true value. Nevertheless, we collocate $\boldsymbol{x}_{j}$ only at the element mid points and not with the element end points. Therefore, we can safely ignore the effects of linearizing the EB on $B_{j i}$.

\section{Fast Multipole Method}

Here, we describe FMM for two dimensional homogeneous problems (Eq. (4)). Let $N$ be the number of grid points inside the Cartesian domain $\Omega_{c}$ and $M$ be the number of boundary elements on $\Gamma$. Computation of the boundary integrals in Eq. (15) and (16) require $O\left(M^{2}\right)$ and $O(M N)$ operations, respectively. FMM reduces this operation count to $O(m M)$ and $O(m N)$, respectively, by trading accuracy which is controlled by the number of terms in multipole/local expansions $m$ with $m \ll M$. Typically, $M=O\left(N^{1 / 2}\right)$ in 2D which leads to an overall complexity of $O(N)$. We solve Eq. (15) iteratively through a restarted GMRES during which FMM is employed for one time evaluation of known terms and repetitive evaluation of unknown terms until convergence.

The key idea of FMM is to split interactions between source nodes $\left(\boldsymbol{x}_{i} \in \Gamma_{i}\right)$ and target nodes $\left(\boldsymbol{x}_{j}\right)$ in Eq. (15), (16) into near and far field interactions. While near field interactions are evaluated directly, far field interactions are evaluated by transferring node-node interactions to interactions among clusters of nodes. Typically, nodes are clustered into a hierarchy of bins through a quadtree datastructure (see Fig. 3) as described below.

\subsection{Adaptive quadtree data structure}

An adaptive quadtree data structure $(Q)$ is employed in FMM to facilitate efficient interactions among clusters of nodes by identifying near/far field sets. The quadtree is formed by first identifying a square large enough to contain $\Omega$ (thereby all source and target nodes) and let us refer it as a bin of level 0 . Next, this parent bin is equally divided into four child bins of level 1 . We continue this procedure recursively, i.e., a parent bin at level $l$ is equally divided into four child bins of level $l+1$. The recursive division of a bin is terminated if (a) it contains less than or equal to a 
preset number $(s)$ of source nodes, say, bin capacity, or (b) maximum level $\left(l_{\max }\right)$ of the tree (equivalently, minimum bin size) is reached, or (c) none of the neighbor bins (including self) contain source nodes.

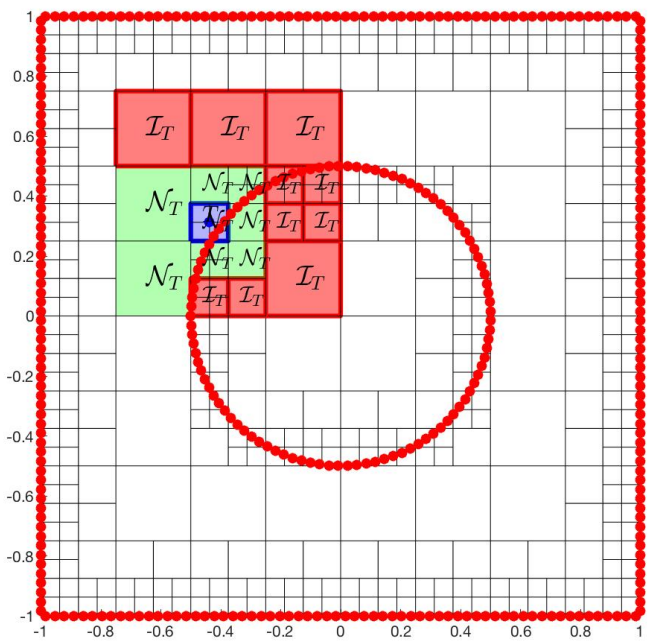

(a)

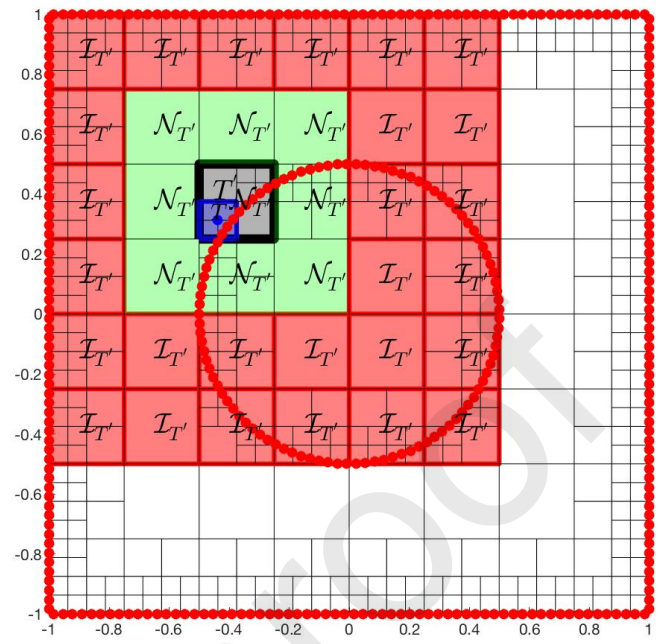

(b)

Fig. 3: Neighbor $(\mathcal{N})$ and interaction $(\mathcal{I})$ lists in the adaptive quadtree data structure $Q$ for (a) bin $T$ (highlighted in blue) and (b) its parent bin $T^{\prime}$ (highlighted in black). For target nodes in the bin $T$, bins in the list $\mathcal{N}_{T}$ contain near field source nodes and bins in the list $\mathcal{I}_{T}$ contain far field source nodes. Filled circles denote centers of boundary elements. Bin capacity $s=2$.

Fig. 3 illustrates $Q$ with an example of a circle $\left(\Gamma_{e b}\right)$ embedded inside a square $\left(\Omega_{c}\right)$ and bin capacity $s=2$. Here, $\Gamma=\Gamma_{c} \cup \Gamma_{e b}$ and centers of the boundary elements $\Gamma_{i}$ (also source nodes) are marked by filled circles. Note that each bin has number of source nodes less than or equal to $s$ due to the stopping criterion (a) above. Furthermore, the bins are finer (larger level $l$ ) near $\Gamma$ and coarser inside $\Omega$ (smaller level $l$ ) owing to the distribution of source nodes $\left(\boldsymbol{x}_{i} \in \Gamma\right.$ ).

Now, consider a bin $T$ (blue square in Fig. 3(a,b)) of level $l$ with side $a$ and the following definitions. Parent of $T$ is the bin on level $l-1$ that contains $T$ and denoted as $T^{\prime}$ (thick black square in Fig. 3(b)). Children $\left(C_{T}\right)$ of $T$ is the set of bins whose parent is $T$ (the four small bins inside $T$ in Fig. 3(a,b)). Bins that do not have children are leaf bins. A bin is said to be well separated from $T$, if it is separated by a distance at least $a$. Next, consider the following lists.

- Neighbors $\left(\mathcal{N}_{T}\right)$ of $T$ is the set of (1) bins at level $l$ that touch $T$ including $T$ itself (smaller green squares in Fig. 3(a)) and (2) leaf bins at coarser levels (with side $>a$ ) that touch $T$ (larger green squares in Fig. 3(a)). For target nodes $\boldsymbol{x}_{j} \in T$, source nodes $\boldsymbol{x}_{i} \in \mathcal{N}_{T}$ constitute the near field set. Neighbors $\left(\mathcal{N}_{T^{\prime}}\right)$ of $T^{\prime}$ are highlighted by the green squares in Fig. 3(b).

- Interaction list $\left(\mathcal{I}_{T}\right)$ of $T$ is the set including (1) children of the bins in $\mathcal{N}_{T^{\prime}}$ that are not in $\mathcal{N}_{T}$ (smaller red squares in Fig. 3(a)) and (2) leaf bins in $\mathcal{N}_{T^{\prime}}$ that are not in $\mathcal{N}_{T}$ (larger red squares in Fig. 3(a)). For target nodes $\boldsymbol{x}_{j} \in T$, source nodes $\boldsymbol{x}_{i} \in \mathcal{I}_{T}$ constitute a far field set. Though bins outside $\mathcal{I}_{T}$, for example $\mathcal{I}_{T^{\prime}}$ (red squares in Fig. 3(b)), are well separated from $T$, they are also well separated from its parent $T^{\prime}$ and the far field interactions involving these bins take place preferentially at the coarser level $l-1$ through $T^{\prime}$.

Note that bins without source nodes (boundary elements) in $\mathcal{N}_{T}, \mathcal{I}_{T}$ are not required and hence ignored. Each bin in $Q$ is tagged with an universal tree index based on Z-order space filling curve and its neighbors (constituting list $\mathcal{N}$ ) are identified efficiently via binary representation of the universal index and bit interleaving/deinterleaving (see $§ 5.3$, Gumerov and Duraiswami (2004)).

Due to different spatial distribution and density of target nodes $\boldsymbol{x}_{j}$ in Eq. (15) with $j=1,2, \ldots, M$ and Eq. (16) with $j=1,2, \ldots, N$, we use two different adaptive data structures, one for solving Eq. (15) for the boundary unknowns and other for interior field evaluation through Eq. (16). Let us denote these with $Q_{M}^{M}$ (Fig. 4(a)) and $Q_{N}^{M}$ (Fig. 4(b)), respectively. Here, the superscript indicates number of source nodes and the subscript indicates number of target nodes. For optimum performance, we use an additional criterion (which overrides condition (a) above) for $Q_{N}^{M}$ in 


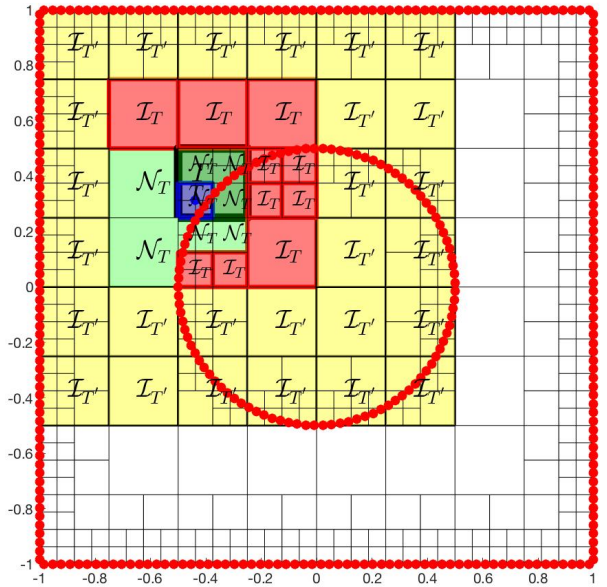

(a)

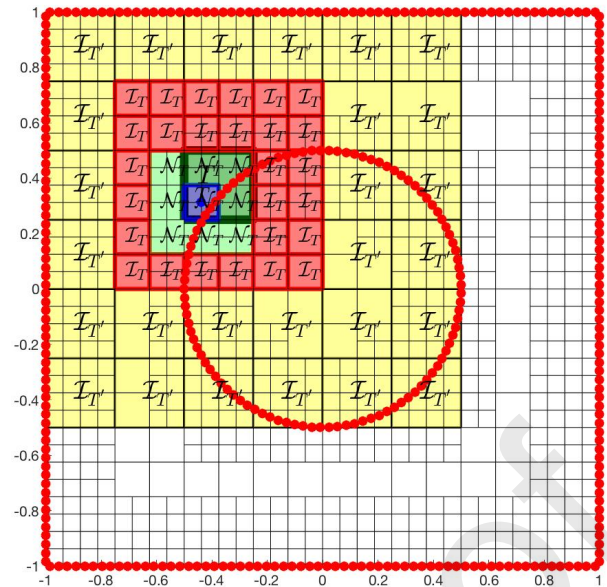

(b)

Fig. 4: Adaptive quadtree data structures used in FMM for a square domain $\left(\Omega_{c}\right)$ with a circle $\left(\Gamma_{e b}\right)$ embedded in it: (a) $Q_{M}^{M}$ for solving Eq. (15) with target nodes $\boldsymbol{x}_{j}$ on $\Gamma$, (b) $Q_{N}^{M}$ for evaluating Eq. (16) with target nodes $\boldsymbol{x}_{j}$ uniformly distributed over $\Omega_{c}$. Here, the boundary elements $\left(\Gamma_{i}\right)$ are marked by filled circles at their centers and bin capacity is 2 . For a reference bin $T$ highlighted in blue, its parent bin $T^{\prime}$ is the thick black square, bins in the neighbor list $\left(\mathcal{N}_{T}\right)$ are the green squares, bins in the interaction list $\left(\mathcal{I}_{T}\right)$ are the red squares. Further, bins in the interaction list of its parent bin $\left(\mathcal{I}_{T^{\prime}}\right)$ are the yellow squares.

which a bin $T$ of level $l<l_{\max }$ must be divided if any of the neighbor bins $\left(\mathcal{N}_{T}\right)$ of level $l+1$ contains source nodes (boundary elements). This criterion reduces the number of direct (near field) interactions from the list $\mathcal{N}$ which are expensive and increases the number of far field interactions from the list $\mathcal{I}$. Furthermore, different bin capacities are used for $Q_{M}^{M}$ and $Q_{N}^{M}$ to account for different target densities (nodes per unit area). The adaptive strategy described above effectively takes advantage of the fact that sources for homogeneous problem lie only on $\Gamma$. Fig. 4(a),(b) exemplifies the data structures $Q_{M}^{M}, Q_{N}^{M}$ for a square domain with a circle embedded inside it. For a bin $T$ highlighted in blue, its parent $T^{\prime}$ (thick black square), its neighbor list $\mathcal{N}_{T}$ (green squares), its interaction list $\mathcal{I}_{T}$ (red squares) and the interaction list of its parent $I_{T^{\prime}}$ (yellow squares) are highlighted.

Fig. 5 depicts various stages in far field interactions between clusters of target nodes $\boldsymbol{x}_{j} \in T$ and source nodes $\boldsymbol{x}_{i} \in \tau$. Here, the source nodes $\boldsymbol{x}_{i}$ lie in a leaf bin $\tau$ with center $z_{c}$ whose parent is $\tau^{\prime}$ with center $z_{c^{\prime}}$. The target nodes $\boldsymbol{x}_{j}$ lie in a leaf bin $T$ with center $z_{L}$ whose parent is $T^{\prime}$ with center $z_{L^{\prime}}$. Let us say $\tau$ and $T$ have sides $a$ which implies that their parents $\tau^{\prime}$ and $T^{\prime}$ have sides $2 a$. Now, consider $\tau^{\prime} \in \mathcal{I}_{T^{\prime}}$ which implies that $\tau^{\prime}$ is at least a distance $2 a$ away from $T^{\prime}$ (i.e., well separated) as shown in Fig. 5. First, sources at all nodes inside $\tau$ including $\boldsymbol{x}_{i}$ are transferred to multipole expansions $M_{k}\left(z_{c}\right)$ at the center of $\tau$ through P2M kernel (see Sec. 4.2). Then, multipole expansions

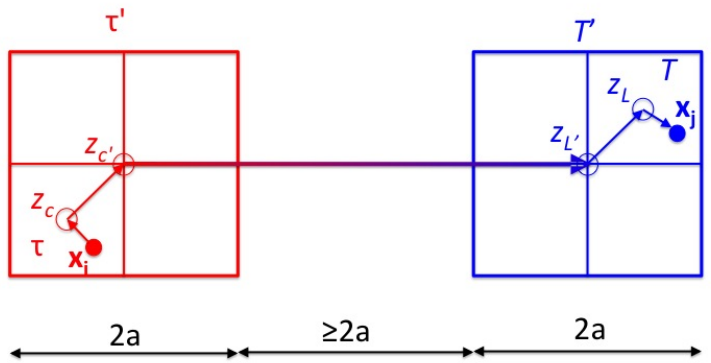

Fig. 5: Schematic of various stages involved in far field interaction between source node $\boldsymbol{x}_{i}$ and target node $\boldsymbol{x}_{j}$ : P2M $\left(\boldsymbol{x}_{i} \rightarrow z_{c}\right)$, M2M $\left(z_{c} \rightarrow z_{c^{\prime}}\right)$, M2L $\left(z_{c^{\prime}} \rightarrow z_{L^{\prime}}\right)$, L2L $\left(z_{L^{\prime}} \rightarrow z_{L}\right)$, L2P $\left(z_{L} \rightarrow \boldsymbol{x}_{j}\right)$. Here, $\boldsymbol{x}_{i}$ lies in a leaf bin $\tau$ with center $z_{c}$ whose parent is $\tau^{\prime}$ with center $z_{c^{\prime}}$ and $\boldsymbol{x}_{j}$ lies in a leaf bin $T$ with center $z_{L}$ whose parent is $T^{\prime}$ with center $z_{L^{\prime}}$. Bins $\tau^{\prime}$ and $T^{\prime}$ of size $2 a$ are well separated if the distance between them is at least $2 a$. Source bins/nodes are in red and target bins/nodes are in blue. 
$M_{k}\left(z_{c^{\prime}}\right)$ at the center of $\tau^{\prime}$ are computed by grouping those from its children including $\tau$ via M2M kernel. Next, multipole expansions at $z_{c^{\prime}}$ are transferred to local expansions $L_{l}\left(z_{L^{\prime}}\right)$ at the center of $T^{\prime}$ through M2L kernel. Then, local expansions at the center of $T^{\prime}$ are translated to centers of its children including $T$ through L2L kernel. Finally, local expansions $L_{l}\left(z_{L}\right)$ at the center of $T$ are used to evaluate the boundary integrals due to far away sources at all target nodes in $T$ including $\boldsymbol{x}_{j}$ through L2P kernel.

Though bins $\tau$ and $T$ in Fig. 5 are well separated from each other, they interact indirectly through their parents (via additional steps M2M, L2L) because interactions over larger clusters are more efficient. So, interactions occur preferentially at larger bins if the source and the target are significantly far away and at smaller bins if the source and the target are somewhat closer but still well separated. The quadtree data structure facilitates this through interaction list $\mathcal{I}$. For example, see $\mathcal{I}_{T^{\prime}}$ (yellow squares) for the bin $T^{\prime}$ (thick black square) and $\mathcal{I}_{T}$ (red squares) for the bin $T$ (blue square) in Fig. 4(a,b).

\subsection{Multipole and local expansion kernels}

FMM in 2D utilizes a complex notation where each physical point $\boldsymbol{x}=(x, y)$ is represented by a point $z=x+\mathrm{i} y$ in the complex plane and the kernels in Eq. (13),(14) are represented in complex form as $G\left(\boldsymbol{x}_{j}, \boldsymbol{x}\right)=\mathfrak{R}\left\{G\left(z_{j}, z\right)\right\}$ and $F\left(\boldsymbol{x}_{j}, \boldsymbol{x}\right) \equiv \partial G\left(\boldsymbol{x}_{j}, \boldsymbol{x}\right) / \partial n=\mathfrak{R}\left\{F\left(z_{j}, z\right)\right\}$ where,

$$
\begin{aligned}
G\left(z_{j}, z\right) & =-\frac{1}{2 \pi} \ln \left(z_{j}-z\right), \\
F\left(z_{j}, z\right) & =n(z) G^{\prime}\left(z_{j}, z\right), \quad \text { with } \quad G^{\prime}\left(z_{j}, z\right) \equiv \frac{\partial G\left(z_{j}, z\right)}{\partial z} \text { and } n(z)=n_{x}+\mathrm{i} n_{y} .
\end{aligned}
$$

The following multipole/local expansion kernels are taken from Liu and Nishimura (2006).

$$
I_{k}(z) \equiv \frac{z^{k}}{k !} \text { for } k \geq 0, \quad O_{k}(z) \equiv \frac{(k-1) !}{z^{k}} \text { for } k \geq 1, \quad \text { and } \quad O_{0}(z) \equiv-\ln (z) .
$$

\section{Multipole expansion (P2M)}

Referring to Fig. 5, let a set of boundary elements $\Gamma_{i}$ have their center inside $\tau\left(\Gamma_{i} \in \tau\right)$ and constitute a part $S_{0}$ of the boundary $\Gamma$. The boundary integrals over $S_{0}$ with kernels $G\left(z_{j}, z\right)$ and $F\left(z_{j}, z\right)$ are expressed in terms of multipole expansions $M_{k}\left(z_{c}\right)$ at the center of $\tau$ as,

$$
\int_{S_{0}} G\left(z_{j}, z\right) q(z) \mathrm{d} S(z)=\frac{1}{2 \pi} \sum_{k=0}^{m} O_{k}\left(z_{j}-z_{c}\right) M_{k}\left(z_{c}\right), \quad \int_{S_{0}} F\left(z_{j}, z\right) p(z) \mathrm{d} S(z)=\frac{1}{2 \pi} \sum_{k=1}^{m} O_{k}\left(z_{j}-z_{c}\right) N_{k}\left(z_{c}\right) .
$$

For a linear element $\Gamma_{i}$, the unit normal is constant, say, $n(z)=n_{i}$, and with constant element approximation we have,

$$
\begin{aligned}
M_{k}\left(z_{c}\right) & \equiv \int_{S_{0}} I_{k}\left(z-z_{c}\right) q(z) \mathrm{d} S(z)=\sum_{\Gamma_{i} \in \tau} q_{i} \int_{\Gamma_{i}} I_{k}\left(z-z_{c}\right) \mathrm{d} S(z)=\sum_{\Gamma_{i} \in \tau} R_{k i}^{(\tau)} q_{i}, \\
N_{k}\left(z_{c}\right) & \equiv \int_{S_{0}} n(z) I_{k-1}\left(z-z_{c}\right) p(z) \mathrm{d} S(z)=\sum_{\Gamma_{i} \in \tau} p_{i} n_{i} \int_{\Gamma_{i}} I_{k-1}\left(z-z_{c}\right) \mathrm{d} S(z)=\sum_{\Gamma_{i} \in \tau} T_{k i}^{(\tau)} p_{i} .
\end{aligned}
$$

where, $R_{k i}^{(\tau)} \equiv \int_{\Gamma_{i}} I_{k}\left(z-z_{c}\right) \mathrm{d} S(z)=\left(\frac{\left(z_{i+1}-z_{c}\right)^{k+1}-\left(z_{i}-z_{c}\right)^{k+1}}{(k+1) !}\right)\left(\frac{z_{i+1}^{*}-z_{i}^{*}}{\left|z_{i+1}-z_{i}\right|}\right)$, and $T_{k i}^{(\tau)}=n_{i} R_{k-1 i}^{(\tau)}$. Here, multipole expansions are truncated to $m+1$ terms. Note that $N_{k}\left(z_{c}\right)$ are needed only for $k \geq 1$, so we set $N_{0}\left(z_{c}\right)=0$.

\section{Moment-to-moment translation (M2M)}

Referring to Fig. 5, multipole expansions at the center of $\tau^{\prime}$ are expressed in terms of those at the center of $\tau$ as,

$$
M_{k}\left(z_{c^{\prime}}\right) \equiv \int_{S_{0}} I_{k}\left(z-z_{c^{\prime}}\right) q(z) \mathrm{d} S(z)=\sum_{l=0}^{k} I_{k-l}\left(z_{c}-z_{c^{\prime}}\right) M_{l}\left(z_{c}\right) .
$$


Moment-to-local translation (M2L)

Referring to Fig. 5, let the bin $T$ (and thereby $T^{\prime}$ ) contain a set of target nodes $\boldsymbol{x}_{j}$ ( $z_{j}$ in complex form). Local expansions $L_{l}\left(z_{L^{\prime}}\right)$ at the center of $T^{\prime}$ are expressed in terms of multipole expansions $M_{k}\left(z_{c^{\prime}}\right)$ at the center of $\tau^{\prime}$ as,

$$
\int_{S_{0}} G\left(z_{j}, z\right) q(z) \mathrm{d} S(z)=\sum_{l=0}^{m} L_{l}\left(z_{L^{\prime}}\right) I_{l}\left(z_{j}-z_{L^{\prime}}\right), \quad L_{l}\left(z_{L^{\prime}}\right)=\frac{(-1)^{l}}{2 \pi} \sum_{k=0}^{m} O_{l+k}\left(z_{L^{\prime}}-z_{c^{\prime}}\right) M_{k}\left(z_{c^{\prime}}\right) .
$$

\section{Local-to-local translation ( L2L)}

Referring to Fig. 5, local expansions at the center of $T$ are translated from those at the center of its parent $T^{\prime}$ as,

$$
L_{l}\left(z_{L}\right)=\sum_{k=0}^{m-l} I_{k}\left(z_{L}-z_{L^{\prime}}\right) L_{l+k}\left(z_{L^{\prime}}\right)
$$

\section{Local-to-particle translation ( $L 2 P)$}

Finally, referring to Fig. 5, the boundary integral at a set of target nodes $\boldsymbol{x}_{j}$ due to far away source elements $\Gamma_{i} \in \tau$ constituting $S_{0}$ are evaluated through local expansions at the center of $T$ as,

$$
\int_{S_{0}} G\left(\boldsymbol{x}_{j}, \boldsymbol{x}\right) q(\boldsymbol{x}) \mathrm{d} S(\boldsymbol{x})=\mathfrak{R}\left\{\int_{S_{0}} G\left(z_{j}, z\right) q(z) \mathrm{d} S(z)\right\}=\mathfrak{R}\left\{\sum_{l=0}^{m} L_{l}\left(z_{L}\right) I_{l}\left(z_{j}-z_{L}\right)\right\} .
$$

Note that the translations M2M, M2L, L2L, L2P are applicable for the kernel $F\left(z_{j}, z\right)\left(\right.$ with $\left.N_{0}\left(z_{c}\right)=0\right)$ and are not repeated here. The approximation error in multipole expansions truncated to $m+1$ terms scales as $e_{m} \sim\left(\frac{z_{i}-z_{c^{\prime}}}{z_{j}-z_{c^{\prime}}}\right)^{m+1} \leq$ $\left(\frac{\sqrt{2} a}{3 a}\right)^{m+1}$ (see Fig. 5). This yields $m \sim-3.06 \log _{10} e_{m}-1$. For example, to have the approximation error $e_{m}$ below $O\left(10^{-10}\right)$, we consider at least 31 terms in multipole/local expansions.

\subsection{Traversal through the quadtree data structure}

Far field interactions in FMM are efficiently computed via upward and downward traversal through a quadtree data structure $Q$ (Carrier et al., 1988).

\subsubsection{Upward traversal}

First, boundary sources are transferred to multipole expansions at centers of the leaf bins that contain them (P2M) by using Eq. (26), (27). Then, multipole expansions of their parent bins are computed by summing the moments via M2M translations from the four children by using Eq. (28). This procedure is carried through the hierarchy up to level two.

\subsubsection{Downward traversal}

Starting with bins $\left(T^{\prime}\right)$ in level two, contribution of far away sources from bins in their interaction list $\left(\tau^{\prime} \in \mathcal{I}_{T^{\prime}}\right)$ is transferred to local expansions $L_{l}\left(z_{L^{\prime}}\right)$ at their centers via M2L translations by using Eq. (29) which are then transferred to their children $(T)$ via L2L translations by using Eq. (30). This procedure is continued through the hierarchy down to the level of leaf bins. For bins $T$ of level $l>2$, the local expansions via L2L translations from their parent bins $T^{\prime}$ are added to the local expansions via M2L translation from bins in their interaction list $\left(\tau \in \mathcal{I}_{T}\right)$. Once the leaf bins $T$ are encountered, far away source contributions are evaluated at each target node inside them by using L2P translation (Eq. 31). Finally, contribution of nearby sources from neighbor bins $\mathcal{N}_{T}$ are evaluated directly through Eq. (20), (21) and added to the contribution of far away sources evaluated through L2P translation (Eq. 31).

\subsubsection{Complexity estimates}

Complexity estimates of FMM to solve Eq. (15) for the boundary unknowns through the quadtree $Q_{M}^{M}$ is given by (Nishimura, 2002),

$$
Q_{M}^{M},\left(\boldsymbol{x}_{i}, \boldsymbol{x}_{j} \in \Gamma\right):[\underbrace{O(m M)}_{P 2 M}+\underbrace{O\left(m^{2} M / s\right)}_{M 2 M}+\underbrace{O\left(27 m^{2} M / s\right)}_{M 2 L}+\underbrace{O\left(m^{2} M / s\right)}_{L 2 L}+\underbrace{O(m M)}_{L 2 P}+\underbrace{O(9 s M)}_{P 2 P(\text { direct })}] \times n_{G M R E S,}
$$


where, $M$ is the number of boundary elements, $m$ is the number of terms in multipole/local expansions, $s$ is the bin capacity (maximum number of source nodes inside a leaf bin) and $n_{\text {GMRES }}$ is the number of iterations in GMRES to solve Eq. (15). Here, number of leaf bins is $O(M / s)$. For constant $m$ and $s$, the total complexity is $O(M)$ (Nishimura, 2002). Once $m=-3.06 \log _{10} e_{m}-1$ is fixed based on accuracy requirements, optimum value of $s$ that balances the dominant operations M2L and P2P is given by $s=\sigma m$, where $\sigma$ depends on the average number of bins in the interaction list that contain non-zero source nodes (maximum 27, see Fig. 4(a) for example) and the work required per each direct interaction between a source and a target. The latter can be decreased significantly by precomputing and storing the influence coefficients (IC) for each bin $\tau\left(A_{j i}, B_{j i}, \forall \boldsymbol{x}_{j} \in \tau, \boldsymbol{x}_{i} \in \mathcal{N}_{\tau}\right)$ which need to be computed only once. We exploit these features to enhance performance during the iterative solution via GMRES.

Complexity estimates of FMM to evaluate Eq. (16) for the interior solution through the quadtree $Q_{N}^{M}$ and the domain integral in Eq. (B.2) through the quadtree $Q_{N}^{N}$ are given by,

$$
\begin{aligned}
Q_{N}^{M},\left(\boldsymbol{x}_{i} \in \Gamma, \boldsymbol{x}_{j} \in \Omega\right) & : \underbrace{O(m M)}_{P 2 M}+\underbrace{O\left(m^{2} M / s\right)}_{M 2 M}+\underbrace{O\left(27 m^{2} N / s^{2}\right)}_{M 2 L}+\underbrace{O\left(m^{2} N / s^{2}\right)}_{L 2 L}+\underbrace{O(m N)}_{L 2 P}+\underbrace{O\left(9 s^{2} M\right)}_{P 2 P \text { (direct) }}, \\
Q_{N}^{N},\left(\boldsymbol{x}_{i}, \boldsymbol{x}_{j} \in \Omega\right) & : \underbrace{O(m N)}_{P 2 M}+\underbrace{O\left(m^{2} N / s\right)}_{M 2 M}+\underbrace{O\left(27 m^{2} N / s\right)}_{M 2 L}+\underbrace{O\left(m^{2} N / s\right)}_{L 2 L}+\underbrace{O(m N)}_{L 2 P}+\underbrace{O(9 s N)}_{P 2 P \text { (direct) }},
\end{aligned}
$$

where, $N$ is the number of interior nodes. For $Q_{N}^{M}$, each leaf bin has a maximum of $s$ source nodes $\left(\boldsymbol{x}_{i}\right)$ and $O\left(s^{2}\right) \operatorname{target}$ nodes $\left(\boldsymbol{x}_{j}\right)$. While M2M operation involves $O(M / s)$ bins (leaf bins for source nodes), the operations M2L and L2L involve $O\left(N / s^{2}\right)$ bins (leaf bins for target nodes). The direct (P2P) interactions involve $O(M / s)$ bins (the bins $\tau$ closer to $\Gamma$ with non-zero source nodes inside $\mathcal{N}_{\tau}$, see Fig. 4(b) for example) each with $O\left(s^{2} \times 9 s\right)$ operations. Bins with empty neighbor list sense the boundary sources via M2L and L2L operations. Optimum choice of $s$ is proportional to $m$ with a overall complexity of $O(m N)+O\left(m^{2} M\right)$ which implies $O(M)$ complexity for small $N$ and $O(N)$ complexity for large $N$. For $Q_{N}^{N}$, the complexity estimates are similar to $Q_{M}^{M}$ except that the number of source/target nodes is $N$ instead of $M$, resulting in a overall complexity of $O(m N)$.

\subsection{Preconditioner}

A block diagonal (left) pre-conditioner is employed to improve convergence of the iterative solver (GMRES). Self-interactions of leaf bins (i.e., direct evaluations among all sources and targets inside a bin) constitute a block diagonal matrix. We invert this matrix and pass it as a left-preconditioner to GMRES solver. For a fixed tolerance, we observed $20-50 \%$ reduction in the total number of iterations depending on the case and mesh size. Further, the preconditioner is more effective at smaller mesh sizes for a fixed bin capacity (Table 1-3) as the sparsity of the block diagonal matrix increases with increasing mesh size. For a given mesh, the preconditioner is more effective with larger bin capacity $s$ (fewer levels in $Q_{M}^{M}$ ) due to reduced sparsity of the block diagonal matrix.

\section{Results}

The Method of Manufactured Solutions (MMS) is a powerful and widely used technique to test the accuracy of a solver in which an exact (particular) solution is manufactured to satisfy a set of governing equations that are altered through forcing terms. While testing a solver via MMS, boundary conditions are typically derived from the exact (particular) solution which leads to a trivial homogeneous solution and nullifies the boundary effects on performance. Therefore, MMS is not necessarily a suitable technique to test the effect of boundary conditions on the performance.

Here, we present test cases with a non-trivial homogeneous solution that challenge the performance of a solver in the presence of boundary effects. This is achieved by either vanishing source terms in part of the Cartesian domain ( $\Omega_{c}^{i}$ for exterior problems and $\Omega_{c}^{e}$ for interior problems) or choosing boundary conditions that are different from the particular (inhomogeneous) solution. In the following examples, the Cartesian domain is discretized uniformly with $N$ grid points and spacing $\Delta x, \Delta y$ along $x, y$ - directions, respectively. Note, however, that the method is applicable to non-uniform grids as well. The boundary mesh used in BEM has $M$ elements with size $\Delta l$.

FMM performance (compute time) shown in the following sections depends on the ratio of far field to near field (direct) interactions and the optimum ratio in turn depends on the number of multipole/local expansions (determined by the accuracy requirements) and EB geometry (i.e., source distribution along $\Gamma$ ). We observe that downward traversal (M2L+L2L) and local (L2P+direct) operations take significantly (an order of magnitude) larger time than other operations such as upward traversal $(\mathrm{P} 2 \mathrm{M}+\mathrm{M} 2 \mathrm{M})$. We choose the bin capacity $s$ that minimizes compute time for the downward traversal and local operations. 


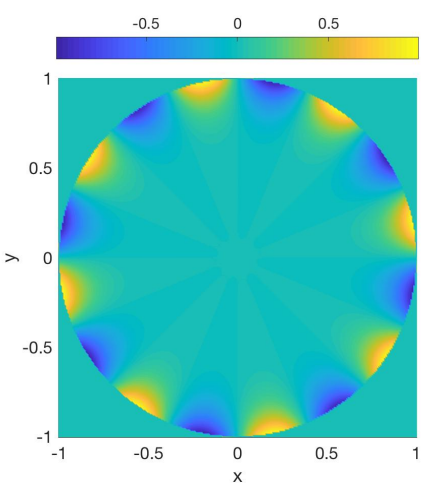

(a)

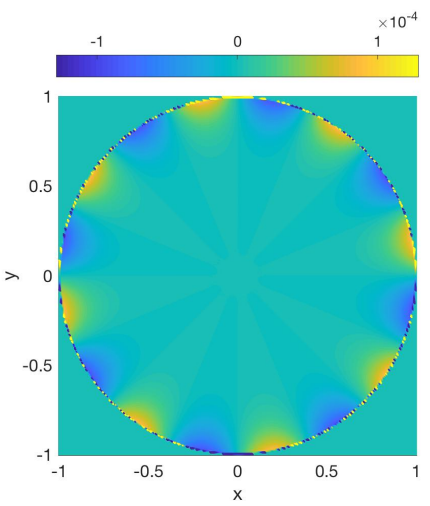

(b)

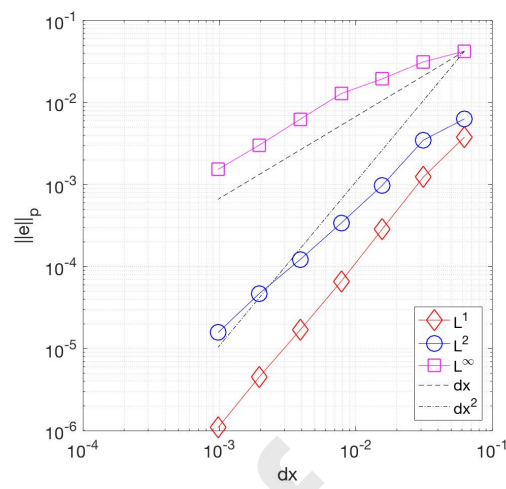

(c)

Fig. 6: The unit circle case with $S=0$ : (a) homogeneous solution through FMM, (b) error distribution for $N=256^{2}$, and (c) grid convergence.

\subsection{The unit circle with a homogeneous source}

Here, we consider a homogeneous problem $(S=0)$ with $\Omega$ corresponding to interior of the unit circle $\left(\Gamma=\Gamma_{e b}\right)$. Since the particular solution is trivial $\left(p^{i}=0\right)$, this case specifically targets accuracy of the BEM/FMM solver by solving for $p^{h}$ and comparing it with an exact solution given below,

$$
\begin{aligned}
p(\theta) & =\sin (n \theta) \quad \text { on } \quad \Gamma, \\
p(r, \theta) & =r^{n} \sin (n \theta) .
\end{aligned}
$$

Here, $n=8$ is chosen. The Cartesian domain $\left(\Omega_{c}\right)$ and $\Gamma$ are uniformly discretized with $\Delta x=\Delta y=\Delta l$. Fig. 6(a) shows the homogeneous solution $\left(p^{h}\right)$ obtained via FMM for $N=256^{2}$ uniform grid and spatial distribution of corresponding error is shown in Fig. 6(b). The grid convergence shown in Fig. 6(c) indicates a first order accuracy in local $\left(L^{\infty}\right)$ norm and second order accuracy in global $\left(L^{1}, L^{2}\right)$ norms.

\subsection{Circle embedded in a square domain}

Consider a square domain $[-1,1]^{2}$ with a circle of radius $R=0.5$ centered at the origin embedded in it and $\Omega=\Omega_{c}^{e}$. Let us consider Neumann boundary condition $\frac{\partial p}{\partial n}=q$ on $\Gamma_{e b}$ and a source term $S(r, \theta)$ as in Eq. (37).

$$
\begin{array}{lll}
S(r, \theta)=D\left(k^{2}-n^{2}\right) r^{(k-2)} \sin (n \theta), & \frac{\partial p}{\partial n}=q \quad \text { on } \Gamma_{e b}, \\
p^{i}(r, \theta)=D r^{k} \sin (n \theta), & p^{h}(r, \theta)=-D\left(\frac{k}{n}\right)\left(\frac{r}{R}\right)^{(n-k)} r^{k} \sin (n \theta)+R q \ln r .
\end{array}
$$

Here, $D=(\sqrt{2})^{-\max (k, n)}$ is a normalization constant and we choose $k=1, n=-5, q=1$. The particular $\left(p^{i}\right)$ and homogeneous $\left(p^{h}\right)$ solutions given in Eq. (38) are used to prescribe Dirichlet boundary conditions on the Cartesian boundary $\left(\Gamma_{c}\right)$ for the inhomogeneous problem (Eq. (3) solved via GMG) and the homogeneous problem (Eq. (4) solved via BEM/FMM), respectively. For the homogeneous problem, boundary condition on $\Gamma_{e b}$ becomes $\frac{\partial p^{h}}{\partial n}=$ $q-\frac{\partial p^{i}}{\partial n}=q-D k R^{(k-1)} \sin (n \theta)$. The exact solution is $p(r, \theta)=p^{i}(r, \theta)+p^{h}(r, \theta)$. For BEM, both $\Gamma_{c}$ and $\Gamma_{e b}$ are uniformly discretized into straight elements with $\Delta l=\Delta x=\Delta y=2 / N^{1 / 2}$. In FMM, the multipole/local expansions are truncated to $m=15$ terms.

Fig. 7(a-c) show the numerical solutions obtained through GMG $\left(p^{i}\right), \operatorname{BEM} / \mathrm{FMM}\left(p^{h}\right)$ and their sum for $N=$ $256^{2}$, respectively. Fig. 7 (d) compares profiles along the diagonal from $(-1,-1)$ to $(1,1)$ of the numerical solutions $p^{i}$, $p^{h}$ and their sum $(p)$ with the exact solutions (Eq. 38) $p_{\text {exact }}^{i}, p_{\text {exact }}^{h}$ and $p_{\text {exact }}$, respectively. Note that the region $-0.5<$ $r<0.5$ lies outside $\Omega$ and is excluded. Spatial distribution of error in the numerical solution $(p)$ in $\Omega$ is shown in Fig. 7 (e) for $N=256^{2}$. The error is quantified in terms of the integral $p$-norm defined as $L^{p}=\left(\Delta x \Delta y \sum_{i} \sum_{j}\left|e_{i j}\right|^{p}\right)^{1 / p}$. Grid convergence results in Fig. 7(f) show that the error is locally first order $\left(L^{\infty} \sim O(\Delta x)\right)$ and globally second order $\left(L^{1}, L^{2} \sim O\left(\Delta x^{2}\right)\right)$. This is expected as the constant element approximation used in BEM discretization introduces an error of $O(\Delta l)$. 


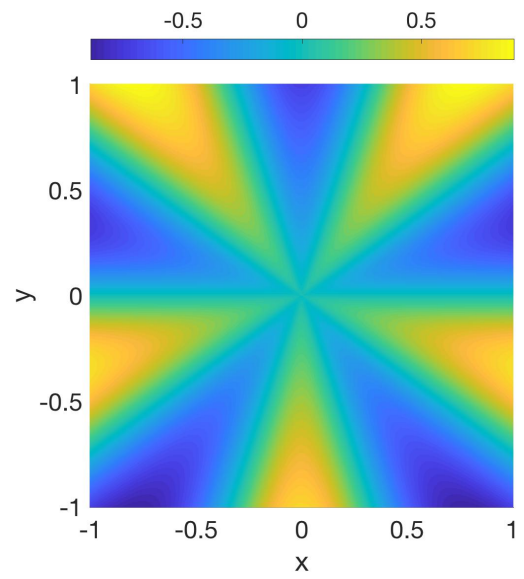

(a)

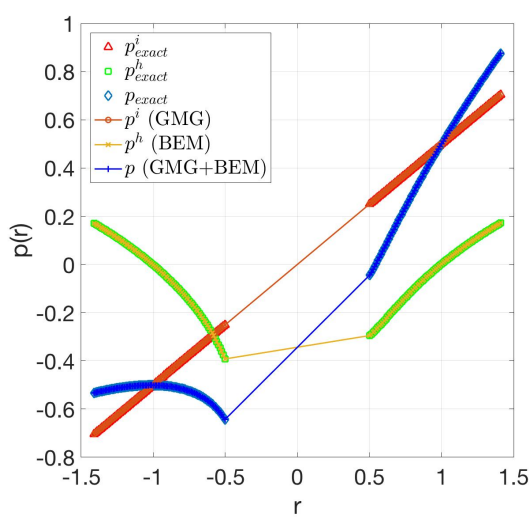

(d)

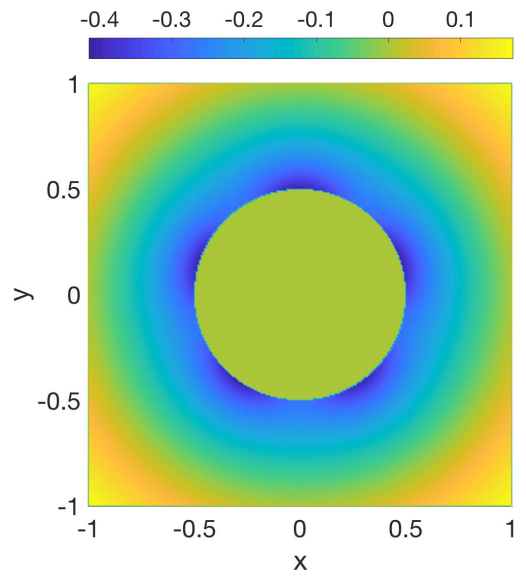

(b)

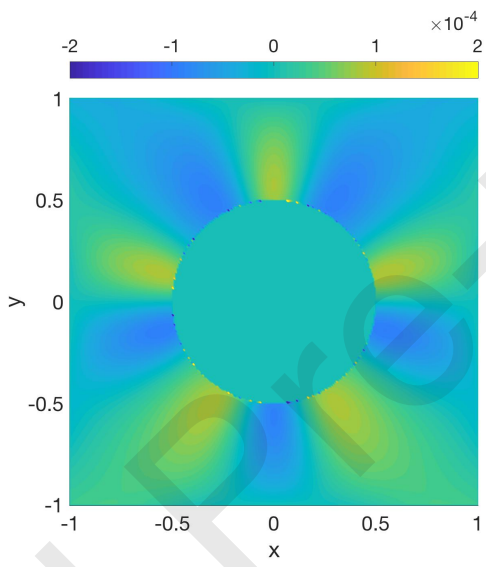

(e)

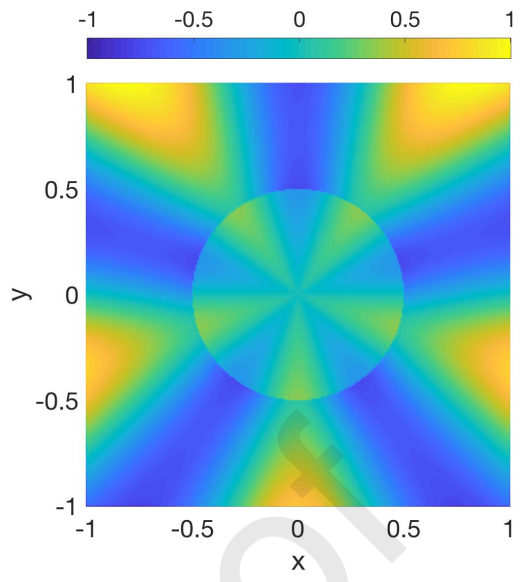

(c)

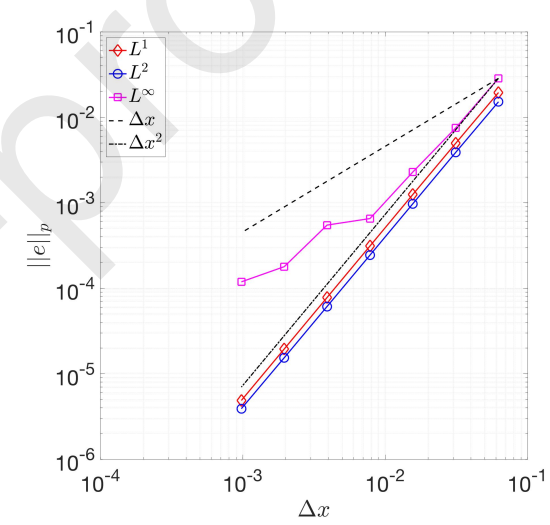

(f)

Fig. 7: The case of circle embedded in a square domain with $S(r, \theta)$ as in Eq. (37): (a) inhomogeneous solution ( $\left.p^{i}\right)$ through GMG without satisfying the EB condition, (b) homogeneous solution $\left(p^{h}\right)$ through BEM/FMM, and (c) combined (GMG+BEM) solution satisfying the EB condition, (d) profiles of numerical and exact solutions $\left(p^{i}, p^{h}, p\right)$ along the diagonal from $(-1,1)$ to $(1,1)$, (e) spatial distribution of the error in the combined solution $(p)$ for $N=256^{2}$, and (f) convergence of the error. Here, multipole/local expansions in FMM are truncated to $m=15$ terms.

Table 1 shows the compute time (on a MacBook Pro with $2.5 \mathrm{GHz}$ Intel Core i7 processor and $16 \mathrm{~GB} / 1600 \mathrm{MHz}$ DDR3 memory) taken by GMG, BEM, FMM, and a stand-alone FMM described in Appendix B along with individual contributions from each part in the solution algorithm. For GMG, we report compute time for two different problems: EB-GMG is a stand-alone method which solves the original undivided problem, Eq. (1)-(2), through an immersed boundary method (Rapaka and Sarkar, 2016; Rapaka and Samtaney, 2018) in which the solution at ghost nodes (nodes outside $\Omega$ that are immediate neighbors to at least one node inside $\Omega$ ) is reconstructed via bilinear approximation (Sec. 2.1) by imposing boundary condition $\left(\frac{\partial p}{\partial n}=q\right)$ on $\Gamma_{e b}$ only at the finest multi-grid level. GMG $\left(\Omega_{c}\right)$ solves the inhomogeneous problem, Eq. (3), in the Cartesian domain $\Omega_{c}$ and ignores the EB so that the boundary condition is not satisfied on $\Gamma_{e b}\left(\frac{\partial p}{\partial n} \neq q\right)$. Our tests indicate that convergence of EB-GMG for this case is optimum when the coarsest level has four points (i.e., the number of multigrid levels is one less than the ideal). Notably, Coco and Russo (2013); Crockett et al. (2011) also found it necessary to sacrifice few coarse grid levels for convergence of GMG involving EB geometries.

For BEM/FMM, compute times to solve Eq. (15) for unknowns on $\Gamma$ via GMRES and evaluate Eq. (16) for interior $(\Omega)$ solution are reported in Table 1 along the columns GMRES and Eval, respectively. Time taken to compute the influence coefficients $A, B$ in Eq. (15) for BEM is shown in the column IC. In FMM, $A, B$ are computed only for near field (direct) interactions and far field interactions are evaluated through multipole and local expansions. In 


\begin{tabular}{|c|c|c|c|c|c|c|c|c|c|c|c|c|c|c|c|c|c|}
\hline \multicolumn{2}{|c|}{ Grid } & EB-GMG & $\operatorname{GMG}\left(\Omega_{c}\right)$ & \multicolumn{3}{|c|}{ Time for BEM } & \multicolumn{6}{|c|}{ Time for FMM } & GMG+FMM & \multicolumn{3}{|c|}{ Time for stand-alone FMM } & $s$ \\
\hline$N$ & $M$ & Time (Iter) & Time (Iter) & IC & GMRES & Eval & IC & RHS & GMRES (Iter) & MLE & Eval & Total & Time & $p^{i}$ & $p^{h}$ (Iter) & Total & $Q_{M}^{M}$ \\
\hline $64^{2}$ & 357 & $0.11(12)$ & $0.06(7)$ & 0.02 & 0.05 & 0.13 & 0.06 & 0.01 & $0.04(20)$ & 0.01 & 0.04 & 0.16 & 0.22 & 0.14 & $0.18(20)$ & 0.32 & 16 \\
\hline $128^{2}$ & 714 & 0.30 (16) & $0.13(7)$ & 0.07 & 0.05 & 0.80 & 0.08 & 0.01 & $0.06(20)$ & 0.03 & 0.10 & 0.28 & 0.41 & 0.54 & $0.33(22)$ & 0.87 & 32 \\
\hline $256^{2}$ & 1427 & $0.70(16)$ & $0.26(8)$ & 0.22 & 0.08 & 6.48 & 0.12 & 0.01 & $0.07(20)$ & 0.05 & 0.19 & 0.44 & 0.70 & 1.61 & $0.55(22)$ & 2.16 & 64 \\
\hline $512^{2}$ & 2853 & $\mathbf{3 . 5 0}(20)$ & $0.51(8)$ & 0.81 & 0.18 & 50.81 & 0.29 & 0.02 & $0.10(22)$ & 0.13 & 0.42 & 0.97 & 1.48 & 5.78 & $1.03(24)$ & 6.81 & 128 \\
\hline $1024^{2}$ & 5705 & $24.03(22)$ & $1.92(8)$ & 3.09 & 0.95 & 405.48 & 0.35 & 0.03 & $0.26(28)$ & 0.23 & 0.83 & 1.70 & 3.62 & 22.80 & $1.97(31)$ & 24.77 & 128 \\
\hline $2048^{2}$ & 11409 & $118.19(24)$ & $9.06(8)$ & 12.99 & 4.27 & - & 0.84 & 0.05 & $0.70(35)$ & 0.46 & 1.81 & 3.86 & 12.92 & 93.46 & $4.42(37)$ & 97.88 & 128 \\
\hline
\end{tabular}

Table 1: Performance of GMG, BEM and FMM for the case of circle embedded in a square domain. Here, EB-GMG is a stand-alone method for complex geometries that solves Eq. (1)-(2) and imposes boundary conditions on $\Gamma_{e b}$ only at the finest grid level via bilinear reconstruction Eq. (5-6); GMG $\left(\Omega_{c}\right)$ solves Eq. (3) in the Cartesian domain $\Omega_{c}$ and ignores the EB. Here, $L_{x}=L_{y}=2, D=1, \Delta x=\Delta y=\Delta l$. Convergence criterion for GMG and GMRES: normalized residual $\leq 10^{-8}$. Iter - number of iterations in GMG/GMRES, IC - influence coefficients $(A, B)$ for direct interactions, MLE - multipole and local expansions. Bin capacity $s$ (number of source nodes per bin) is shown for the quadtree $Q_{M}^{M}$ and $s=16$ for both $Q_{N}^{N}$ and $Q_{N}^{M}$. Note: the maximum number of target nodes for the smallest leaf bins (near $\Gamma$ ) of $Q_{N}^{M}$ is $s^{2}$. All times are in seconds.

FMM, right hand side (including known terms) of Eq. (15) is computed only once (column RHS in Table 1) while the left hand side (including unknown terms) of Eq. (15) is repeatedly evaluated using an iterative solution till GMRES converges (column GMRES). Number of iterations for GMRES are shown in column Iter. Column MLE shows wall time for computing multipole and local expansion coefficients involving P2M, M2M, M2L, L2L kernels. Column Eval shows wall time for L2P and P2P (direct) interactions. Bin capacity for the quadtrees are reported in columns $s$. For $Q_{N}^{M}$, the smallest leaf bins near $\Gamma$ each have a maximum number of target nodes $s^{2}$. For the stand-alone FMM, compute times for obtaining the particular solution $\left(p^{i}\right)$ involving domain integrals and the homogeneous solution $\left(p^{h}\right)$ involving boundary integrals are shown separately. Also, number of iterations taken by GMRES solver is reported as Iter.

Our convergence criteria for GMG is that the normalized residual be less than or equal to $10^{-8}$. While the $\operatorname{GMG}\left(\Omega_{c}\right)$ has an ideal performance with compute time proportional to $N$, EB-GMG has poor performance with a worsening (non-linear) dependency of compute time on $N$. The loss of linear complexity can be attributed to sacrificing coarser multigrid levels necessary for EB applications. Though EB-GMG has simplified treatment of EB (only at finest multi-grid level), it serves to demonstrate the loss of linear complexity which is found even in more sophisticated approaches involving multi-level representation of EB (Coco and Russo, 2013; Crockett et al., 2011). While the regular BEM has $O\left(N^{3 / 2}\right)$ complexity $\left(O(N)\right.$ for GMRES and $O\left(N^{3 / 2}\right)$ for $\left.E v a l\right)$, the FMM for homogeneous problem shows $O(M)$ complexity in the column Total which implies that $O\left(m^{2} M\right)$ dominates $O(m N)$ for the range of mesh sizes considered (see Sec. 4.3.3 for complexity estimates). Overall, compute times of GMG+FMM (column highlighted in bold in Table 1) are an order of magnitude lower than that of stand-alone FMM (Total column highlighted in bold in Table 1). For example, compute time of GMG+FMM for $N=2048^{2}$ is $12.98 s$ while that of the stand-alone FMM is 96.43s. The difference is chiefly due to differences in computation of the inhomogeneous part $\left(p^{i}\right)$ : column $\operatorname{GMG}\left(\Omega_{c}\right)$ takes $9.02 s$ while column $p^{i}$ of stand-alone FMM takes $90.24 s$. This shows the efficiency of GMG compared with that of FMM for inhomogeneous problems. Note, however, that the compute time of FMM for the homogeneous problem $(3.86 s)$ is similar to that of the GMG for the inhomogeneous problem $(9.06 s)$.

\subsection{Stand-alone GMG vs. FMM}

In this section we compare stand alone performance of GMG and FMM with the source term $S=1$ whose particular solution is given below. Here, a square domain is considered for two reasons: 1) complex geometries require the domain $\Omega$ to be discretized into non-Cartesian cells (e.g., triangles in 2D) which evades the purpose of EB methods, and 2) scope of the analytical expressions derived in Appendix B to compute $G * S$ is limited to the Cartesian cells. Dirichlet boundary conditions are prescribed with values derived from the particular solution.

$$
p(r, \theta)=r^{2} / 4
$$

Table 2 shows compute times and $L^{2}$ norm of the error for two stand-alone methods to solve above problem in Cartesian domain: GMG and FMM. The global error ( $\|e\|_{2}$ in Table 2) is of second order for both GMG and FMM whereas the local error ( $L^{\infty}$ norm, not shown) is of second order for GMG and first order for FMM, as expected. Remarkably, the GMG is an order of magnitude faster than FMM (bold columns in Table 2). For example, GMG takes $8.99 s$ for $N=2048^{2}$ while FMM takes $96.65 s$ of which a majority $93.22 s$ is due to the evaluation of the domain integrals and a negligible $3.43 \mathrm{~s}$ is due to the evaluation of the boundary integrals. 
Journal Pre-proof

\begin{tabular}{|c|c|c|c|c|c|c|c|c|c|c|}
\hline \multicolumn{2}{|c|}{ Grid } & \multicolumn{2}{|c|}{ GMG } & \multicolumn{4}{|c|}{ Time for stand-alone FMM } & \multicolumn{3}{|c|}{ Bin Capacity $(s)$} \\
\hline$N$ & $M$ & Time (Iter) & $\|e\|_{2}$ & $\begin{array}{c}\text { domain integrals } \\
\sum_{i}^{N} C_{j i} S_{i}\end{array}$ & $\begin{array}{l}\text { boundary integrals (Iter) } \\
\alpha p_{j}+\sum_{i}^{M}\left(A_{j i} q_{i}-B_{j i} p_{i}\right)\end{array}$ & Total & $\|e\|_{2}$ & $Q_{N}^{N}$ & $Q_{M}^{M}$ & $Q_{N}^{M}$ \\
\hline $64^{2}$ & 256 & $0.06(8)$ & $1.22 \times 10^{-4}$ & 0.14 & $0.16(9)$ & 0.30 & $2.03 \times 10^{-5}$ & 16 & 16 & 16 \\
\hline $128^{2}$ & 512 & $0.09(8)$ & $3.05 \times 10^{-5}$ & 0.50 & $0.24(10)$ & 0.74 & $5.10 \times 10^{-6}$ & 16 & 32 & 16 \\
\hline $256^{2}$ & 1024 & $0.14(8)$ & $7.63 \times 10^{-6}$ & 1.62 & $0.49(10)$ & 2.11 & $1.28 \times 10^{-6}$ & 16 & 64 & 16 \\
\hline $512^{2}$ & 2048 & $0.46(8)$ & $1.91 \times 10^{-6}$ & 5.79 & $0.77(11)$ & 6.56 & $3.20 \times 10^{-7}$ & 16 & 128 & 16 \\
\hline $1024^{2}$ & 4096 & $\mathbf{1 . 8 3}(8)$ & $4.77 \times 10^{-7}$ & 24.58 & $1.60(18)$ & 26.18 & $8.04 \times 10^{-8}$ & 16 & 128 & 16 \\
\hline $2048^{2}$ & 8192 & 8.99 (8) & $1.19 \times 10^{-7}$ & 93.22 & $3.43(31)$ & 96.65 & $2.07 \times 10^{-8}$ & 16 & 128 & 16 \\
\hline
\end{tabular}

Table 2: Performance of GMG and FMM for the case of a square domain. Here, $L_{x}=L_{y}=2, \Delta x=\Delta y=\Delta l$. Convergence criterion for GMG/GMRES: normalized residual $\leq 10^{-8}$. Iter - number of iterations in GMG/GMRES. Number of terms in multipole and local expansions is 15 . Bin capacity $s$ (of source nodes) is shown for the quadtrees $Q_{N}^{N}, Q_{M}^{M}, Q_{N}^{M}$. Note that for $Q_{N}^{M}$, the maximum number of target nodes inside the smallest leaf bins (near $\Gamma$ ) is $s^{2}$. All times are in seconds.

\begin{tabular}{|c|c|c|c|c|c|c|c|c|c|c|c|c|c|}
\hline \multicolumn{2}{|c|}{ Grid } & \multicolumn{6}{|c|}{ Time for GMG+FMM } & \multicolumn{3}{|c|}{ Time for stand-alone FMM } & \multicolumn{3}{|c|}{ Bin Capacity $(s)$} \\
\hline \multirow{2}{*}{$N$} & \multirow{2}{*}{$M$} & \multirow{2}{*}{$\begin{array}{c}\text { GMG } \\
\text { Time (Iter) }\end{array}$} & \multicolumn{3}{|c|}{ FMM } & \multirow[b]{2}{*}{ Total } & \multirow{2}{*}{ Total } & \multirow{2}{*}{$\begin{array}{c}\text { DI } \\
C * S\end{array}$} & \multirow{2}{*}{$\begin{array}{c}\mathrm{BI}(\text { Iter }) \\
\alpha p+A q-B p\end{array}$} & \multirow{2}{*}{ Total } & \multirow{2}{*}{$Q_{N}^{N}$} & \multirow{2}{*}{$Q_{M}^{M}$} & \multirow{2}{*}{$Q_{N}^{M}$} \\
\hline & & & IC & BS (Iter) & DS & & & & & & & & \\
\hline 64 & 48 & $0.05(8)$ & 0.06 & $0.23(32)$ & 0.08 & 0.38 & 0. & 0.18 & $0.48(32)$ & 0. & 16 & 16 & 32 \\
\hline $128^{8}-x$ & 96 & 8) & 0.06 & $0.25(35)$ & 0.12 & 0.43 & 0.5 & 0.50 & $35)$ & 1.0 & 16 & 32 & 32 \\
\hline $256^{2}$ & 1931 & 8) & 0.14 & $0.31(37)$ & 0.21 & 0.66 & 0.8 & 1.64 & 7) & 2.4 & 16 & 64 & 32 \\
\hline $512^{2}$ & 3861 & $0.46(8)$ & 0.54 & 0.39 (39) & 0.37 & 1.30 & 1.76 & 5.69 & 1.45 (39) & 7.14 & 16 & 128 & 32 \\
\hline $1024^{2}$ & 7721 & $1.93(8)$ & 0.65 & $0.79(45)$ & 0.70 & 2.14 & 4.07 & 23.64 & $2.86(45)$ & 26.50 & 16 & 128 & 32 \\
\hline $2048^{2}$ & 15442 & $9.02(8)$ & 0.90 & $1.70(50)$ & 1.36 & 3.96 & 12.98 & 90.24 & $6.19(50)$ & 96.43 & 16 & 128 & 32 \\
\hline
\end{tabular}

Table 3: Performance of GMG + FMM and stand-alone FMM for the complex geometry case of Askham and Cerfon $(2017)$ with $L_{x}=L_{y}=1, \Delta x=$ $\Delta y \sim 4 \Delta l$. Here, DI - domain integrals associated with the inhomogeneous problem, BI - boundary integrals associated with the homogeneous problem, Iter - number of iterations for GMG/GMRES. Convergence criterion for GMG and GMRES: normalized residual $\leq 10^{-8}$. Number of terms in multipole and local expansions is $\mathrm{m}=15$. Bin capacity $s$ (of source nodes) is shown for the quadtrees $Q_{M}^{M}, Q_{N}^{M}, Q_{N}^{N}$. The smallest leaf bins $\left(\right.$ near $\Gamma$ ) of $Q_{N}^{M}$ each have a maximum number of target nodes $(s / 4)^{2}=64$. All times are in seconds. BS-boundary solution, DS-domain solution, IC-influence coefficients $\left(A_{j i}, B_{j i}\right)$ for direct interactions (source nodes $\left.\boldsymbol{x}_{i} \in \mathcal{N}_{T}\right)$.

\subsection{Multiply connected domain}

Here, we consider an irregular and multiply connected domain (Askham and Cerfon, 2017) defined by two boundaries: $r(\theta)=c_{0}+\sum_{j}\left(c_{j} \cos (j \theta)+d_{j} \sin (j \theta)\right)$. The non-zero coefficients of the outer boundary are $c_{0}=0.25, c_{5}=$ $0.02, c_{6}=c_{8}=c_{10}=d_{3}=0.01$ and the inner boundary are $c_{0}=0.05, c_{2}=c_{5}=c_{7}=d_{3}=0.005$. The source term and exact solution are given by

$$
S(x, y)=-200 \sin (10(x+y))+2, \quad p(x, y)=\sin (10(x+y))+x^{2}-3 y+8 .
$$

Dirichlet boundary condition (Eq. (40)) is prescribed on $\Gamma_{e b}$ for $p$ while $p^{i}=0$ is prescribed on $\Gamma_{c}$ for GMG. The source term is continuous across the EB and is zero for $\psi>0.1$. The inhomogenenous solution $p^{i}$ (Fig. 8(a) for $N=256^{2}$ ) obtained through GMG is interpolated on to $\Gamma_{e b}$ through bicubic approximation (Sec. 2.2) to compute the boundary condition for $p^{h}=p-p^{i}$ on $\Gamma_{e b}$. The homogeneous solution is then obtained via FMM and is shown in Fig. 8(b). Grid convergence reported in Fig. 8(c) confirms second order global accuracy $\left(L^{1}, L^{2}\right)$ and first order local accuracy $\left(L^{\infty}\right)$, as expected. We repeated the simulation with a stand-alone FMM solver wherein the inhomogeneous solution is also obtained through FMM as described in Appendix B.3. Peformance of stand-alone FMM is compared with that of the combined GMG-FMM method in Table. 3. For stand-alone FMM, column BI refers to the time taken by terms involving the boundary integrals (for both boundary solution through GMRES and interior solution, i.e., the homogeneous part) and DI refers to the time taken by the domain integrals (i.e., the inhomogeneous term involving $G * S)$. The combined GMG-FMM $\left(12.98 s\right.$ for $\left.N=2048^{2}\right)$ outperforms the stand-alone FMM (96.43s) by an order of magnitude in terms of the total simulation time. While the time taken by BI in stand-alone FMM is similar to the time taken for the homogeneous solution by GMG+FMM solver, DI in stand-alone FMM takes an order of magnitude larger time to solve the inhomogeneous problem compared with the GMG. This shows the superior computational efficiency of the combined GMG-FMM approach against the stand-alone FMM although both have the linear complexity. 


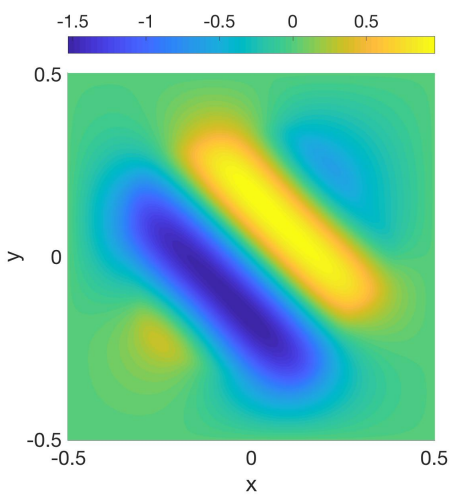

(a)

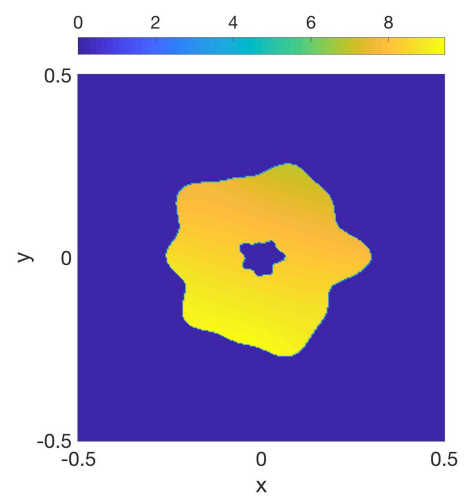

(b)

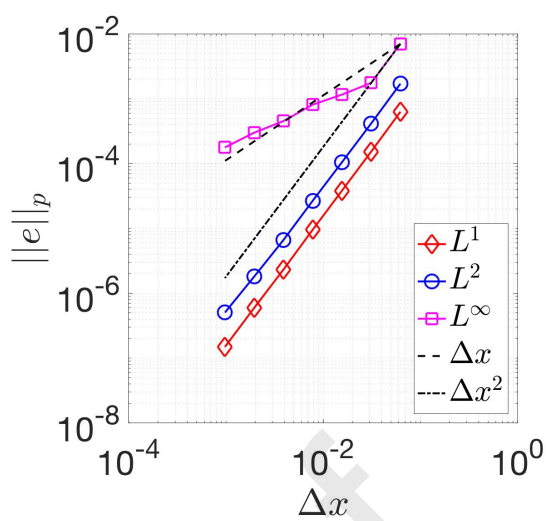

(c)

Fig. 8: Multiply connected domain with $S=0$ for $\psi>0.1$ : (a) inhomogeneous solution through GMG, (b) homogeneous solution through BEM/FMM for $N=256^{2}$, and (c) grid convergence. $\Delta l \approx \Delta x / 4$.

\subsection{Four-lobe structure}

Here, we consider the domain to be the region between the unit square and a complex (EB) surface defined by $r(\theta)=R_{m}+R_{d} \cos (4 \theta)$ with $R_{m}=0.305, R_{d}=0.117$. The source term considered and corresponding exact solution are given below (Hosseinverdi and Fasel, 2018),

$$
\begin{aligned}
& S(r, \theta)=12\left(10 r^{2}-1\right) e^{-10 r^{2}}+\sum_{k=1}^{4} 40\left(10 r_{k}^{2}-1\right), \\
& p(r, \theta)=0.3 e^{-10 r^{2}}+\sum_{k=1}^{4} e^{-10 r_{k}^{2}}, \quad r_{k}=\sqrt{(x \pm 0.45)^{2}+(y \pm 0.45)^{2}} .
\end{aligned}
$$

Dirichlet boundary conditions are prescribed on all boundaries using Eq. (42). The source term is continuous across the EB $(\psi=0)$ but zero outside $\Omega$ for $\psi>0.1\left(\in \Omega_{c}^{i}\right)$ so as to ensure a non-trivial homogeneous solution and that the GMG solver alone does not yield the total solution $(p)$.

Fig. 9(a) shows the inhomogeneous solution $\left(p^{i}\right)$ for $N=256^{2}$ uniform grid obtained via GMG without considering the effects of EB. Then, $p^{i}$ is interpolated on to $\Gamma_{e b}$ via bilinear approximation (Eq. (5)) to compute the boundary condition for the homogeneous solution as $p^{h}=p-p^{i}$. Here, $\Gamma_{e b}$ is discretized into $4 N^{1 / 2}$ elements with uniform angular resolution. A homogeneous solution, shown in Fig. 9(b), is then obtained via FMM with $P=35$ terms in multipole/local expansions. The combined solution is then compared with the exact solution shown in Fig. 9(c). The spatial distribution of error is shown in Fig. 9(d) for $N=256^{2}$ and the grid convergence shown in Fig. 9(e) indicates a second order accuracy in both local $\left(L^{\infty}\right)$ and global $\left(L^{1}, L^{2}\right)$ norms.

\subsection{Three-lobe structure: exterior}

Here, we consider the domain $\Omega$ to be the region between the unit square and a complex EB defined by $r(\theta)=$ $R_{m}+R_{d} \cos (3 \theta)$ with $R_{m}=0.55, R_{d}=0.38$. The source term and exact solution are given below (Hosseinverdi and Fasel, 2018),

$$
\begin{aligned}
& S(r, \theta)=7 r^{2} \cos (3 \theta), \\
& p(r, \theta)=r^{4} \cos (3 \theta) .
\end{aligned}
$$

Dirichlet boundary conditions are prescribed on all boundaries using Eq. (44). The source term is continuous across the EB ( $\psi=0$ ) but made zero outside $\Omega$ for $\psi>0.1$ so as to ensure a non-trivial homogeneous solution.

Fig. 10(a) shows the inhomogeneous solution $\left(p^{i}\right)$ for $N=256^{2}$ uniform grid obtained via GMG without considering the effects of EB. Then, $p^{i}$ is interpolated on to $\Gamma_{e b}$ via Eq. (5) to compute the boundary condition for the homogeneous solution as $p^{h}=p-p^{i}$. Here, $\Gamma_{e b}$ is discretized with $4 N^{1 / 2}$ elements of uniform angular resolution. A 


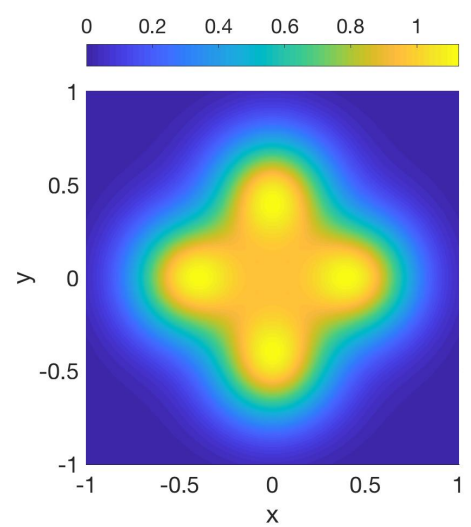

(a)

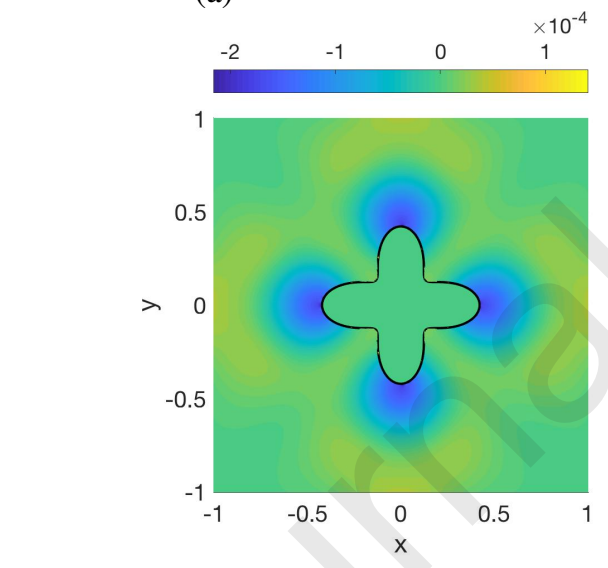

(d)

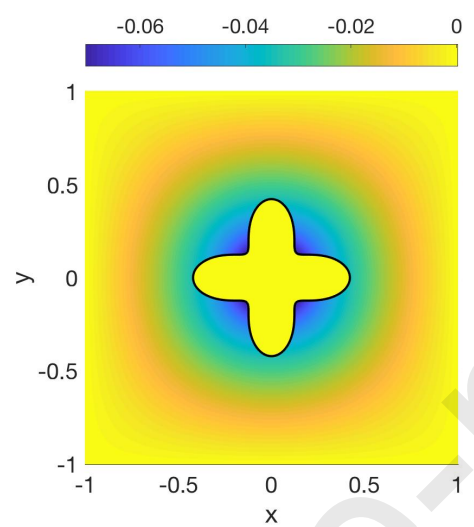

(b)

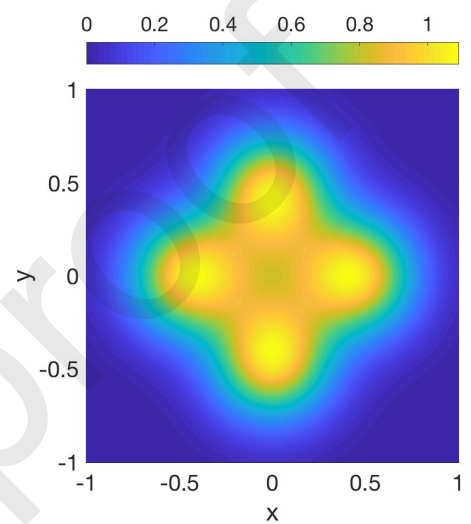

(c)

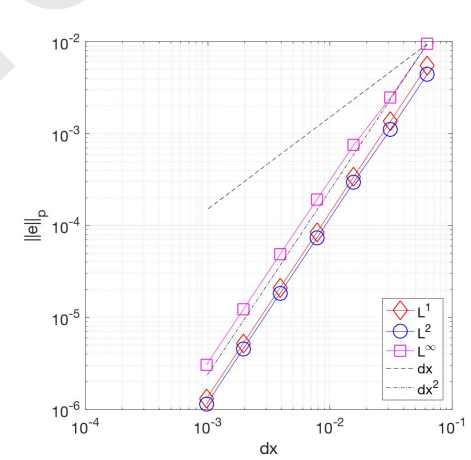

(e)

Fig. 9: (a) Inhomogeneous solution $\left(p^{i}\right)$ through GMG ( $S=0$ for $\left.\psi>0.1\right)$, (b) homogeneous solution $\left(p^{h}\right)$ through FMM, (c) exact solution, (d) distribution of error $\left(N=256^{2}\right)$, and (e) grid convergence for the case four-lobe structure. Source is continuous across EB and zero outside $\Omega$ for $\psi>0.1\left(\in \Omega_{c}^{i}\right)$. 
homogeneous solution, shown in Fig. 10(b), is then obtained via FMM with $P=35$ terms in multipole/local expansions. The combined solution is then compared with the exact solution shown in Fig. 10(c). The spatial distribution of error is shown in Fig. 10(d) for $N=256^{2}$ and the grid convergence shown in Fig. 10(e) indicates first order accuracy in local $\left(L^{\infty}\right)$ norm and second order accuracy in global $\left(L^{1}, L^{2}\right)$ norms. The first order error is due to the constant element approximation used in BEM/FMM.

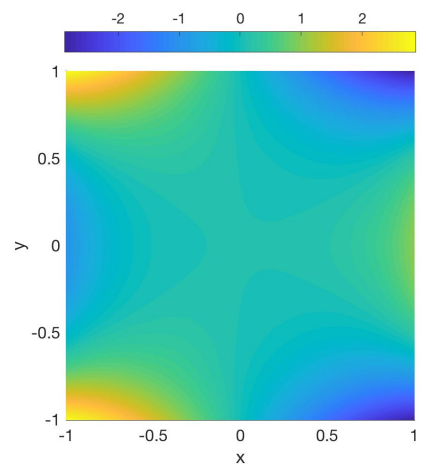

(a)

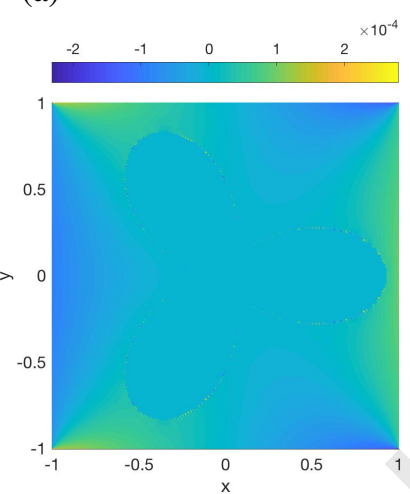

(d)

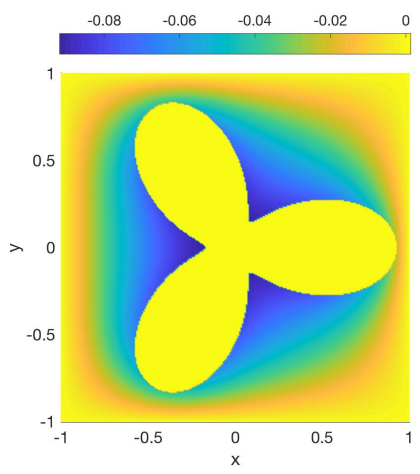

(b)

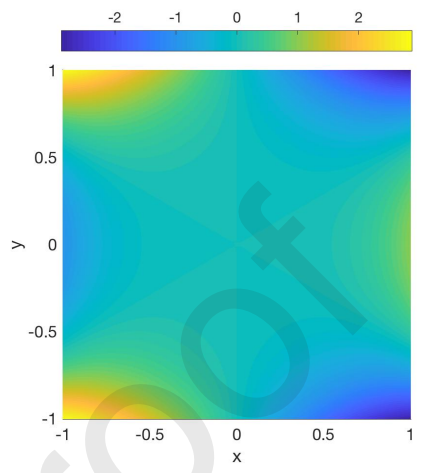

(c)

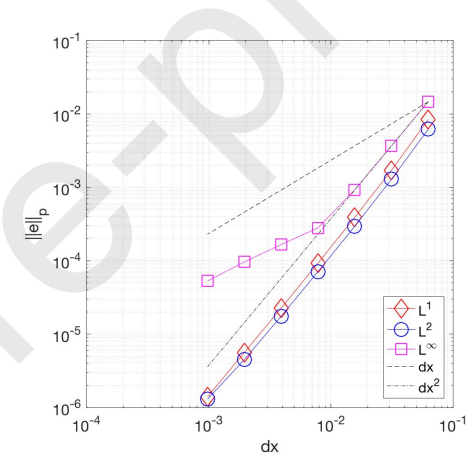

(e)

Fig. 10: (a) Inhomogeneous solution $\left(p^{i}\right)$ through GMG, (b) homogeneous solution $\left(p^{h}\right)$ through FMM, (c) exact solution, (d) distribution of error $\left(N=256^{2}\right)$, and (e) grid convergence for the case three-lobe structure: exterior.

\subsection{Three-lobe structure: interior}

Here, we consider the domain $\Omega$ to be interior of a closed curve (EB) defined by $r(\theta)=R_{m}+R_{d} \cos (3 \theta)$ with $R_{m}=0.55, R_{d}=0.38$. The source term and exact solution are given in Eq. (43) and Eq. (44), respectively. Dirichlet boundary condition is prescribed for $p$ at $\Gamma_{e b}$ using Eq. (44) and $p^{i}=0$ at $\Gamma_{c}$. The source term is continuous across $\Gamma_{e b}(\psi=0)$ but made zero outside $\Omega$ for $\psi>0.1$ to ensure a non-trivial homogeneous solution.

Fig. 11(a) shows the inhomogeneous solution $\left(p^{i}\right)$ obtained via GMG for $N=256^{2}$ uniform grid. Then, the boundary condition for the homogeneous solution $p^{h}=p-p^{i}$ on $\Gamma_{e b}$ is evaluated by interpolating $p^{i}$ on to $\Gamma_{e b}$ from $p^{i}$ at nearby Cartesian nodes via Eq. (5). Here, $\Gamma_{e b}$ is discretized into $4 N^{1 / 2}$ elements of uniform angular resolution. A homogeneous solution, shown in Fig. 11(b), is then obtained via FMM with $P=35$ terms in multipole/local expansions. The combined solution is then compared with the exact solution shown in Fig. 11(c). The spatial distribution of error is shown in Fig. 11(d) for $N=256^{2}$ and the grid convergence shown in Fig. 11(e) indicates first order accuracy in local $\left(L^{\infty}\right)$ norm and second order accuracy in global $\left(L^{1}, L^{2}\right)$ norms. The first order error is due to the constant element approximation used in FMM.

\subsection{Annulus domain}

Here, we consider the domain to be an annulus with inner radius $R_{i}=0.25$ and outer radius $R_{o}=0.75$. 


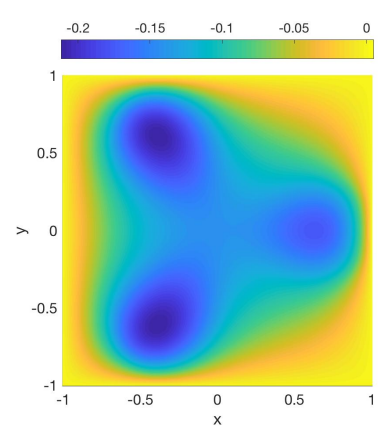

(a)

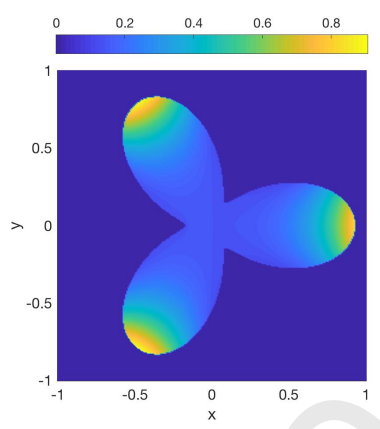

(b)

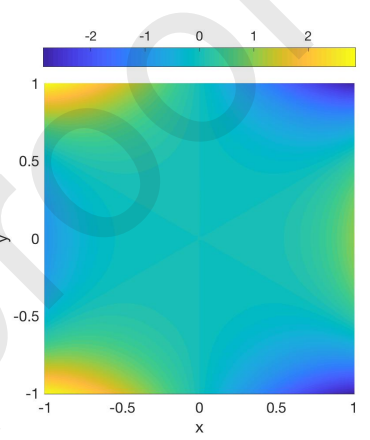

(c)

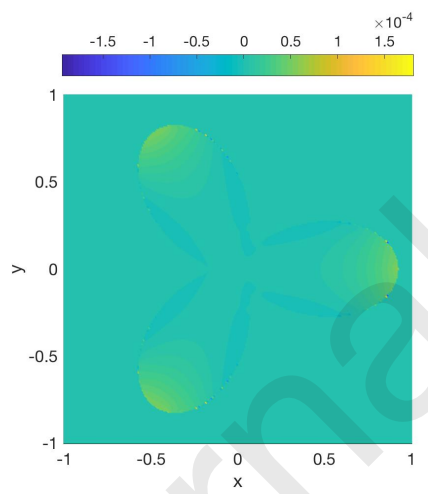

(d)

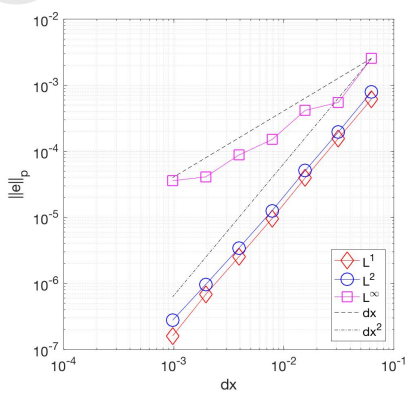

(e)

Fig. 11: (a) Inhomogeneous solution $\left(p^{i}\right)$ through GMG, (b) homogeneous solution $\left(p^{h}\right)$ through FMM, (c) exact solution, (d) distribution of error $\left(N=256^{2}\right)$, and (e) grid convergence for the case three-lobe structure: interior. 


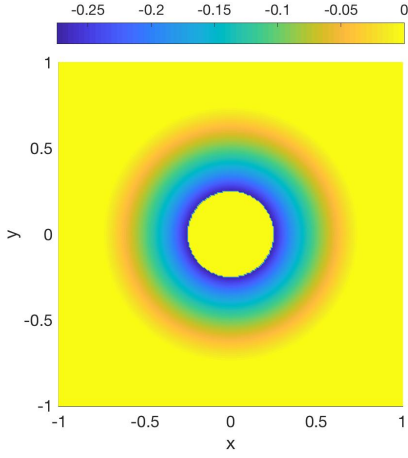

(a)

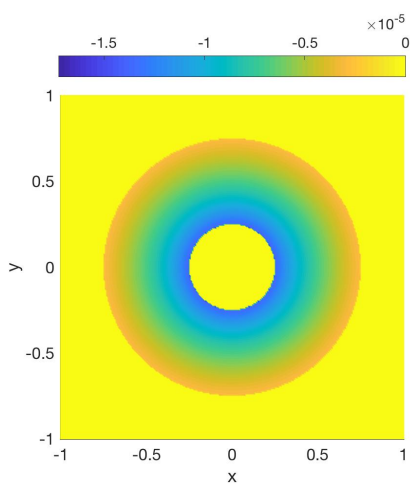

(b)

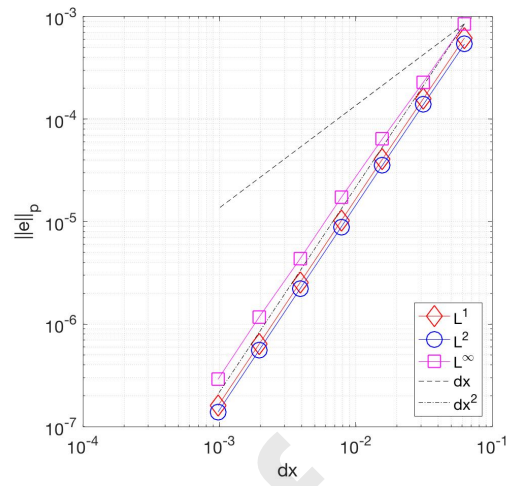

(c)

Fig. 12: (a) Solution ( $p$ ) through FMM, (b) error distribution for $N=256^{2}$, and (c) grid convergence for the annulus domain with $S=0$.

\subsubsection{Homogeneous source}

Here, we consider a homogeneous source $(S=0)$ with Neumann and homogeneous Dirichlet boundary conditions at inner and outer boundaries of the annulus, respectively. The corresponding exact solution varies only along the radial direction and is given below,

$$
\begin{aligned}
\frac{\partial p}{\partial n} & =q \quad \text { at } \quad r=R_{i} \\
p & =0 \quad \text { at } \quad r=R_{o} \\
p(r, \theta) & =R_{i} q \ln \left(\frac{r}{R_{o}}\right) .
\end{aligned}
$$

Here, $q=1$ is chosen and $\Gamma_{e b}$ is discretized uniformly with $\Delta l=\Delta x=\Delta y$ on both inner and outer boundaries of the annulus. Fig. 12(a) shows the homogeneous solution $\left(p^{h}\right)$ for $N=256^{2}$ obtained via FMM with $P=35$ terms in multipole/local expansions which is then compared with the exact solution Eq. (47). The spatial distribution of corresponding error is shown in Fig. 12(b) for $N=256^{2}$ and the grid convergence shown in Fig. 12(c) indicates a second order accuracy in both local $\left(L^{\infty}\right)$ and global $\left(L^{1}, L^{2}\right)$ norms. This is because the constant element approximation does not introduce any error for this case as the solution is uniform along $\Gamma_{e b}$ and the inhomogeneous problem (Eq. (3)) is discretized with the standard second order central difference scheme.

\subsubsection{Inhomogeneous source}

Here, we consider a uniform source with Neumann and homogeneous Dirichlet boundary conditions at inner and outer boundaries of the annulus, respectively. The corresponding exact solution varies only along the radial direction and is given below,

$$
\begin{aligned}
S(r, \theta) & =1 \\
\frac{\partial p}{\partial n} & =q \quad \text { at } \quad r=R_{i} \\
p & =0 \quad \text { at } \quad r=R_{o} \\
p(r, \theta) & =\frac{r^{2}-R_{o}^{2}}{4}+R_{i}\left(q-\frac{R_{i}}{2}\right) \ln \left(\frac{r}{R_{o}}\right) .
\end{aligned}
$$

Here, $q=1$ is chosen. For GMG solver, the particular solution $p^{i}=r^{2} / 4$ is prescribed on $\Gamma_{c}$. Boundary conditions for the homogeneous solution are $p^{h}=-r^{2} / 4$ and $\frac{\partial p^{h}}{\partial r}=q-r / 2$ at $r=R_{o}$ and $r=R_{i}$, respectively. We also tested $p^{h}=-p^{i}$ and $\frac{\partial p^{h}}{\partial r}=q-\frac{\partial p^{i}}{\partial r}$ where, $p^{i}$ and $\frac{\partial p^{i}}{\partial r}$ are numerically evaluated through Eq. (5) and Eq. (6), respectively. Here, $\Gamma_{e b}$ is discretized uniformly with $\Delta l=\Delta x=\Delta y$ on both inner and outer boundaries of the annulus.

Fig. 13(a) shows the inhomogeneous solution $\left(p^{i}\right)$ for $N=256^{2}$ uniform grid obtained via GMG without considering the effects of EB. A homogeneous solution, shown in Fig. 13(b), is then obtained via FMM with $P=35$ terms 
in multipole/local expansions. The combined solution $\left(p^{i}+p^{h}\right.$ is then compared with the exact solution (Eq. (51)) shown in Fig. 13(c). The spatial distribution of error is shown in Fig. 13(d) for $N=256^{2}$ and the grid convergence shown in Fig. 13(e) indicates a second order accuracy in both local $\left(L^{\infty}\right)$ and global $\left(L^{1}, L^{2}\right)$ norms. This is expected because the constant element approximation does not introduce any error as the solution is constant along $\Gamma_{e b}$.

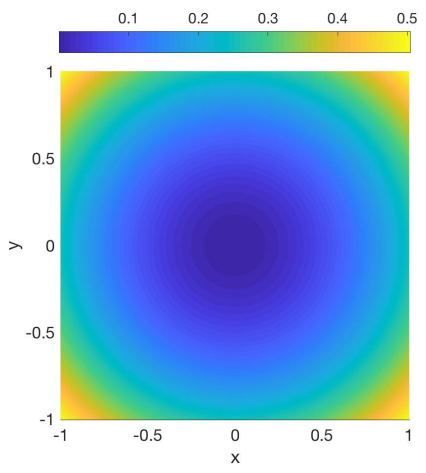

(a)

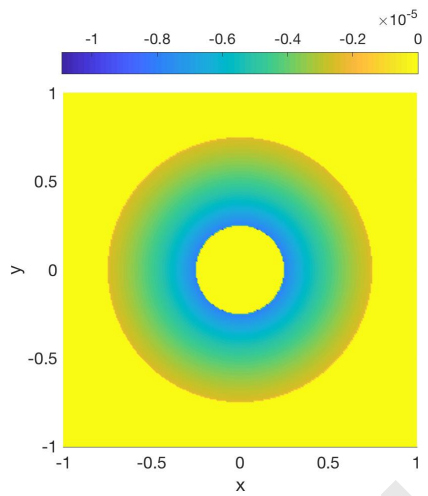

(d)

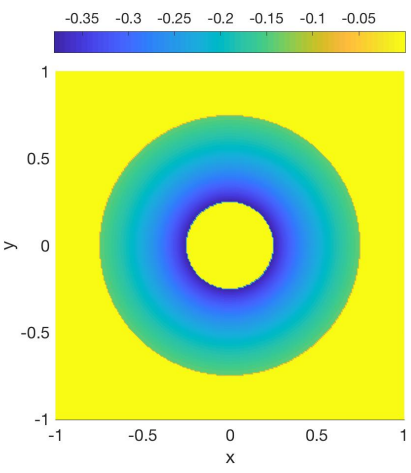

(b)

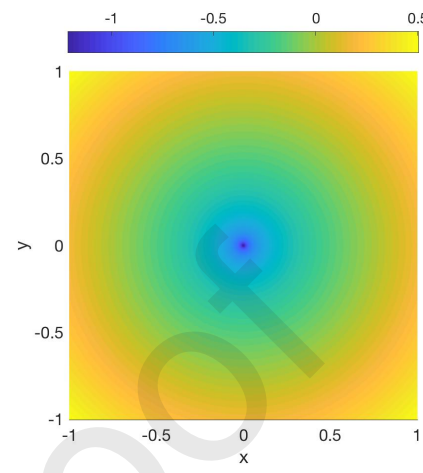

(c)

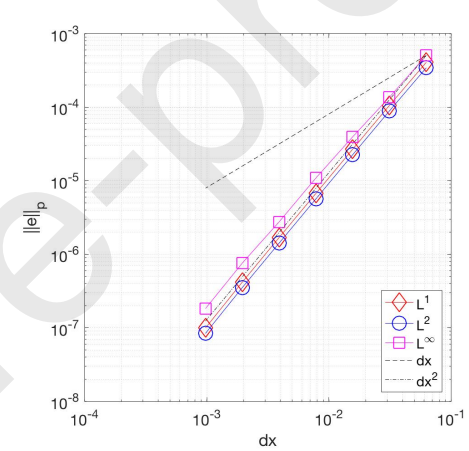

(e)

Fig. 13: Annulus domain with $S=1$ : (a) Inhomogeneous solution $\left(p^{i}\right)$ through GMG without satisfying the EB condition, (b) homogeneous solution $\left(p^{h}\right)$ through FMM satisfying the EB condition, (c) exact solution, (a) distribution of error for $N=256^{2}$, and (b) grid convergence.

\section{Conclusions}

We developed a Poisson solver for complex geometries with linear computational complexity. The method relies on the principle of linear superposition and splits the original problem into an inhomogeneous problem containing sources inside the domain and a homogeneous problem that satisfies appropriate boundary conditions.

While the inhomogeneous problem is solved via a geometric multigrid method (GMG), the homogeneous problem is solved via a boundary element method (BEM). Analytic integration is employed to compute the influence coefficients which provide higher accuracy and significantly faster computation compared with numerical quadratures. Numerical tests show that BEM has $O\left(N^{3 / 2}\right)$ computational complexity in its conventional implementation.

A fast multipole method (FMM) is utilized to compute the boundary integrals in BEM with linear complexity. In FMM, integrals involving near by sources are evaluated directly and far field sources are evaluated by multipole and local expansions. Since the homogeneous problem has sources only on the boundary, adaptive quadtree data structures are employed in FMM. To obtain optimum performance, different adaptive strategies and independent bin capacities/tree depths are used in the quadtree data structures while solving for boundary and interior fields. For the boundary solution, optimum bin capacity is proportional to $N^{1 / 2}$ for large $N$ and for the interior solution, optimum bin capacity is proportional to the number of terms in multipole/local expansions.

A range of test cases are presented including interior/exterior/annulus domains and smooth/sharp geometrical features. Grid convergence studies show that the BEM/FMM, and hence the combined GMG-BEM/FMM solver, 
has first order accuracy in the $\left(L^{\infty}\right)$ norm, and second order accuracy globally ( $L^{1}, L^{2}$ norms), as expected due to the constant element approximation employed in evaluating the boundary integrals. The GMG solver is second order accurate as demonstrated by the tests involving constant solution along the embedded boundaries.

We also tested performance of a stand-alone FMM that solves the original inhomogeneous problem which requires evaluation of the domain integrals in addition to the boundary integrals present in the homogeneous problem. Numerical tests for various cases show that the compute time of the domain integral is an order of magnitude larger compared with that of the boundary integrals. Further, compute time of FMM for the homogeneous problem is similar to that of the GMG for the inhomogeneous problem. As a result, the stand-alone FMM is an order of magnitude slower compared with the combined GMG-FMM method. Thus, we retain the efficiency of GMG and versatility of FMM for complex geomtries.

Also, we tested performance of a stand-alone EB-GMG solver that uses an embedded boundary method for the un-split (original) problem which imposes boundary conditions on complex geometry through bilinear reconstruction at finest grid level only. Numerical experiments suggest that convergence of solution requires limiting the number of coarse grid levels leading to loss of linear complexity. In comparison, GMG-FMM solver has linear complexity and is significantly faster than EB-GMG.

Conventional FMM solvers for the Poisson equation have linear complexity but are known to be significantly slower compared with GMG. The present approach eliminates the domain-domain convolutions required in standalone FMM solvers and the multi-level representation of complex geometries required in stand-alone GMG solvers (e.g., EB-GMG). Overall, the present solver is significantly faster compared with stand alone FMM solvers and achieves performance comparable with that of an ideal GMG method even for complex geometry applications.

\section{Appendix A. Boundary integrals for BEM in 2D}

Recall from Sec. 3 that $l$ is the distance from $\boldsymbol{x}_{m}$ along $\hat{\boldsymbol{t}}$ (Fig. 2(a,b)), $\boldsymbol{x}-\boldsymbol{x}_{j}=a \hat{\boldsymbol{n}}+(b+l) \hat{\boldsymbol{t}}, r^{2}=\left|\boldsymbol{x}-\boldsymbol{x}_{j}\right|^{2}=$ $a^{2}+(b+l)^{2}$ and define $m \equiv b+l$.

$$
\begin{aligned}
\int_{-\Delta l_{i} / 2}^{\Delta l_{i} / 2} \ln \left|\boldsymbol{x}-\boldsymbol{x}_{j}\right| \mathrm{d} l(\boldsymbol{x}) & =\frac{1}{2} \int_{-\Delta l_{i} / 2}^{\Delta l_{i} / 2} \ln \left|a^{2}+(l+b)^{2}\right| \mathrm{d} l(\boldsymbol{x})=\frac{1}{2} \int_{b-\Delta l_{i} / 2}^{b+\Delta l_{i} / 2} \ln \left|a^{2}+m^{2}\right| \mathrm{d} m(\boldsymbol{x}) \\
& =\frac{1}{2}\left[\left(m \ln \left|a^{2}+m^{2}\right|\right)_{b-\Delta l_{i} / 2}^{b+\Delta l_{i} / 2}-\int_{b-\Delta l_{i} / 2}^{b+\Delta l_{i} / 2} \frac{2 m^{2}}{a^{2}+m^{2}} \mathrm{~d} m(\boldsymbol{x})\right], \\
& =\left[\frac{m}{2} \ln \left|a^{2}+m^{2}\right|-m+a \tan ^{-1}\left(\frac{m}{a}\right)\right]_{d}^{c}, \quad \because \frac{m^{2}}{a^{2}+m^{2}}=1-\frac{a^{2}}{a^{2}+m^{2}} \\
& =\frac{c}{2} \ln \left|a^{2}+c^{2}\right|-\frac{d}{2} \ln \left|a^{2}+d^{2}\right|-\Delta l_{i}+a \tan ^{-1}\left(\frac{a \Delta l}{a^{2}+c d}\right), \\
& =c \ln r_{i+1}-d \ln r_{i}-\Delta l_{i}+a \tan ^{-1}\left(\frac{a \Delta l}{a^{2}+c d}\right), \\
& =\Delta l_{i}\left(\ln \left|\Delta l_{i} / 2\right|-1\right), \quad \text { for } a=b=0 \text { or } \boldsymbol{x}_{j}=\boldsymbol{x}_{m}, \text { i.e., } i=j .
\end{aligned}
$$

Since $\hat{\boldsymbol{n}}(\boldsymbol{x}) \cdot\left(\boldsymbol{x}-\boldsymbol{x}_{j}\right)=a, B_{j i}$ is 0 for $a=0\left(\boldsymbol{x}_{j}\right.$ lies on $\Gamma_{i}$ or along the line passing through $\boldsymbol{x}_{i}$ and $\left.\boldsymbol{x}_{i+1}\right)$ and is obtained from the integral below for $a \neq 0$,

$$
\begin{aligned}
\theta_{j i} \equiv \int_{-\Delta l_{i} / 2}^{\Delta l_{i} / 2} \frac{\hat{\boldsymbol{n}}(\boldsymbol{x}) \cdot\left(\boldsymbol{x}-\boldsymbol{x}_{j}\right)}{\left|\boldsymbol{x}-\boldsymbol{x}_{j}\right|^{2}} \mathrm{~d} l(\boldsymbol{x}) & =\int_{b-\Delta l_{i} / 2}^{b+\Delta l_{i} / 2} \frac{a}{a^{2}+m^{2}} \mathrm{~d} m(\boldsymbol{x}) \\
& =\tan ^{-1}\left(\frac{c}{a}\right)-\tan ^{-1}\left(\frac{d}{a}\right) \\
& =\tan ^{-1}\left(\frac{a \Delta l_{i}}{a^{2}+c d}\right) .
\end{aligned}
$$$$
\because \tan ^{-1} x-\tan ^{-1} y=\tan ^{-1} \frac{x-y}{1+x y}
$$

Note that $\theta_{j i}$ is discontinuous as $\boldsymbol{x}_{j}$ approaches the element $\Gamma_{i}$ inside/along the circle centered at $\boldsymbol{x}_{m}$ with radius $\Delta l_{i} / 2$, i.e., $a^{2}+b^{2} \leq\left(\frac{\Delta l_{i}}{2}\right)^{2}$ or $a^{2}+c d \leq 0$ (Fig. 2c). As $\boldsymbol{x}_{j}$ approaches any point on the element $\Gamma_{i}$ along $\hat{\boldsymbol{t}}(a=0)$, $\theta_{j i}=0$. As $\boldsymbol{x}_{j}$ approaches the interior of the element $\Gamma_{i}$ from either side $\left(a \rightarrow 0^{ \pm}\right), \theta_{j i} \rightarrow \pm \pi$. As $\boldsymbol{x}_{j}$ approaches the element end points $(c=0$ or $d=0)$ from either side of $\Gamma_{i}\left(a \rightarrow 0^{ \pm}\right), \theta_{j i} \rightarrow \pm \pi / 2$. 


\section{Appendix B. Stand-alone BEM-FMM for the Poisson equation in 2D}

Green's third identity applied to the Poisson equation Eq. (1) yields (analogue of Eq. (11)),

$$
\alpha p\left(\boldsymbol{x}_{0}\right)=-\int_{\Gamma} G\left(\boldsymbol{x}, \boldsymbol{x}_{0}\right) \frac{\partial p}{\partial n}(\boldsymbol{x}) d \Gamma+\int_{\Gamma}^{P V} p(\boldsymbol{x}) \frac{\partial G\left(\boldsymbol{x}, \boldsymbol{x}_{0}\right)}{\partial n} d \Gamma-\int_{\Omega} G\left(\boldsymbol{x}, \boldsymbol{x}_{0}\right) S(\boldsymbol{x}) d \Omega .
$$

Let us discretize $\Gamma$ into boundary elements $\Gamma_{i}, i=1,2, . ., M$, each of size $\Delta l_{i}$ and $\Omega_{c}$ into Cartesian cells $\Omega_{i}, i=$ $1,2, . ., N$, each of size $\Delta x_{i}, \Delta y_{i}$ in $x, y$ directions, respectively. Let us collocate $\boldsymbol{x}_{0}$ with centers of $\Gamma_{i}$ and $\Omega_{i}$. We make the constant element approximation for the source density $S(\boldsymbol{x})$ to have $S(\boldsymbol{x})=S\left(\boldsymbol{x}_{i}\right) \equiv S_{i}$ for $\boldsymbol{x} \in \Omega_{i}$. Above equations become,

$$
\alpha p\left(\boldsymbol{x}_{j}\right)=-\sum_{i=1}^{M} q_{i} A_{i}\left(\boldsymbol{x}_{j}\right)+\sum_{i=1}^{M} p_{i} B_{i}\left(\boldsymbol{x}_{j}\right)-\sum_{i=1}^{N} S_{i} C_{i}\left(\boldsymbol{x}_{j}\right) .
$$

Here, $C_{j i} \equiv C_{i}\left(\boldsymbol{x}_{j}\right)=\int_{\Omega_{i}} G\left(\boldsymbol{x}, \boldsymbol{x}_{j}\right) d \Omega$. Here, $j=1,2, \ldots, M$ for $\boldsymbol{x}_{j} \in \Gamma$ and $j=1,2, \ldots, N$ for $\boldsymbol{x}_{j} \in \Omega$. The last term in above equation is computed efficiently through FMM using a quadtree datastructure $\left(Q^{S}\right)$ for $\boldsymbol{x}_{j} \in \Gamma$ and $\boldsymbol{x}_{j} \in \Omega$. Near field interactions between sources $\boldsymbol{x}_{i}$ and targets $\boldsymbol{x}_{j}$ are computed directly by analytically evaluating $C_{j i}$ as described in Appendix B.1. Far field interactions are evaluated through upward and downward traversal through the quadtree $Q^{S}$. The translation operators (M2M, M2L, L2L, L2P) described in Sec. 4 are used for the domain integrals as well except for P2M. Notably, the multipole coefficients $M_{k}\left(z_{c}\right)$ involve an area integral over each cell $\Omega_{i}$ as described in Appendix B.2.

\section{Appendix B.1. Domain integrals for BEM/FMM (direct evaluation)}

Let $\boldsymbol{x}_{i}$ be the center of a Cartesian cell $\Omega_{i}\left(\left[x_{i}-\frac{\Delta x_{i}}{2}, x_{i}+\frac{\Delta x_{i}}{2}\right] \times\left[y_{i}-\frac{\Delta y_{i}}{2}, y_{i}+\frac{\Delta y_{i}}{2}\right]\right)$ and $\boldsymbol{x}_{j}$ be the target (collocation) node. Let $\boldsymbol{x}_{i}-\boldsymbol{x}_{j}=a \hat{\mathbf{i}}+b \hat{\mathbf{j}}$ and $\boldsymbol{x}-\boldsymbol{x}_{i}=l \hat{\mathbf{i}}+m \hat{\mathbf{j}}$. We can express the distance vector $\boldsymbol{x}-\boldsymbol{x}_{j}=\left(\boldsymbol{x}-\boldsymbol{x}_{i}\right)+\left(\boldsymbol{x}_{i}-\boldsymbol{x}_{j}\right)=(l+a) \hat{\mathbf{i}}+(m+b) \hat{\mathbf{j}}$ so that $\left|\boldsymbol{x}-\boldsymbol{x}_{j}\right|=\sqrt{(l+a)^{2}+(m+b)^{2}}$. Let us denote $a^{ \pm}=a \pm \frac{\Delta x_{i}}{2}$ and $b^{ \pm}=b \pm \frac{\Delta y_{i}}{2}$ and $\tilde{l}=l+a, \tilde{m}=m+b$.

$$
\begin{aligned}
C_{j i} & =-\frac{1}{2 \pi} \int_{\Omega_{i}} \ln \left|\boldsymbol{x}-\boldsymbol{x}_{j}\right| d \Omega=-\frac{1}{4 \pi} \int_{b^{-}}^{b^{+}} \int_{a^{-}}^{a^{+}} \ln \left(\tilde{l}^{2}+\tilde{m}^{2}\right) d \tilde{l} d \tilde{m} \\
& =-\frac{1}{4 \pi} \int_{b^{-}}^{b^{+}}\left(\left[\tilde{l} \ln \left(\tilde{l}^{2}+\tilde{m}^{2}\right)\right]_{a^{-}}^{a^{+}}-2 \int_{a^{-}}^{a^{+}} \frac{\tilde{l}^{2}}{\tilde{l}^{2}+\tilde{m}^{2}} d \tilde{l}\right) d \tilde{m}, \\
& =-\frac{1}{4 \pi} \int_{b^{-}}^{b^{+}}\left[a^{+} \ln \left(a^{+^{2}}+\tilde{m}^{2}\right)-a^{-} \ln \left(a^{-^{2}}+\tilde{m}^{2}\right)\right] d \tilde{m}+\frac{1}{2 \pi} \int_{a^{-}}^{a^{+}} \tilde{l}\left[\tan ^{-1}\left(\frac{b^{+}}{\tilde{l}}\right)-\tan ^{-1}\left(\frac{b^{-}}{\tilde{l}}\right)\right] d \tilde{l} .
\end{aligned}
$$

By using integration by parts we have,

$$
\begin{gathered}
\int_{b^{-}}^{b^{+}} \ln \left(a^{+^{2}}+\tilde{m}^{2}\right) d \tilde{m}=b^{+} \ln \left(a^{+^{2}}+b^{+^{2}}\right)-b^{-} \ln \left(a^{+^{2}}+b^{-2}\right)-2 \Delta y_{i}+2 a^{+}\left[\tan ^{-1}\left(\frac{b^{+}}{a^{+}}\right)-\tan ^{-1}\left(\frac{b^{-}}{a^{+}}\right)\right], \\
\int_{a^{-}}^{a^{+}} \tilde{l} \tan ^{-1}\left(\frac{b^{+}}{\tilde{l}}\right) d \tilde{l}=\frac{a^{+^{2}}}{2} \tan ^{-1}\left(\frac{b^{+}}{a^{+}}\right)-\frac{a^{-2}}{2} \tan ^{-1}\left(\frac{b^{+}}{a^{-}}\right)+\frac{b^{+} \Delta x_{i}}{2}-\frac{b^{+^{2}}}{2}\left[\tan ^{-1}\left(\frac{a^{+}}{b^{+}}\right)-\tan ^{-1}\left(\frac{a^{-}}{b^{+}}\right)\right] .
\end{gathered}
$$

By substituting above expressions in Eq. (B.3) we have,

$$
C_{j i}=-\frac{1}{2 \pi}\left[f\left(a^{+}, b^{+}\right)+f\left(a^{-}, b^{-}\right)-f\left(a^{+}, b^{-}\right)-f\left(a^{-}, b^{+}\right)-\frac{3 \Delta x_{i} \Delta y_{i}}{2}+g\left(a^{+}, b^{ \pm}\right)-g\left(a^{-}, b^{ \pm}\right)+g\left(b^{+}, a^{ \pm}\right)-g\left(b^{-}, a^{ \pm}\right)\right] .
$$

where, $f(r, s)=\frac{r s}{2} \ln \left(r^{2}+s^{2}\right), g\left(r, s^{ \pm}\right)=\frac{r^{2}}{2}\left[\tan ^{-1}\left(\frac{s^{+}}{r}\right)-\tan ^{-1}\left(\frac{s^{-}}{r}\right)\right]=\frac{r^{2}}{2} \tan ^{-1}\left(\frac{r\left(s^{+}-s^{-}\right)}{r^{2}+s^{+} s^{-}}\right)$. Here, some variables such as $a, b, l, m$ are redefined for exclusive use in this section. Note that Eq. (B.4) is applicable even for $i=j$. 


\section{Appendix B.2. Multipole expansions for domain integrals (P2M)}

Let a set of Cartesian cells $\Omega_{i}$ have their center inside a bin $\tau\left(\Omega_{i} \in \tau\right)$ and constitute a part $\Omega_{0}$ of the Cartesian domain $\Omega_{c}$. We denote center of the cell $\Omega_{i}$ in the complex plane as $z_{i}=x_{i}+\mathrm{i} y_{i}$. Then, we can express a point $z \in \Omega_{i}$ in parametric form as $z=z_{i}+s \frac{\Delta x_{i}}{2}+\mathrm{i} t \frac{\Delta y_{i}}{2}$, where $s, t \in[-1,1]$ are parameters. Let the four corners of $\Omega_{i}$ be $z_{1}, z_{2}, z_{3}, z_{4}$ corresponding to $(s, t)=(-1,-1),(1,-1),(-1,1),(1,1)$, respectively. Note that $z_{2}-z_{1}=\Delta x_{i}, z_{3}-z_{1}=\mathrm{i} \Delta y_{i}$ and $z_{4}=z_{2}+z_{3}-z_{1}$. The domain integral over $\Omega_{0}$ is expressed in terms of the multipole expansions $M_{k}\left(z_{c}\right)$ at the center $z_{c}$ of the bin $\tau$ as,

$$
\int_{\Omega_{0}} G\left(z_{j}, z\right) S(z) d \Omega(z)=\frac{1}{2 \pi} \sum_{k=0}^{m} O_{k}\left(z_{j}-z_{c}\right) M_{k}\left(z_{c}\right), \quad M_{k}\left(z_{c}\right) \equiv \int_{\Omega_{0} \in \tau} I_{k}\left(z-z_{c}\right) S(z) d \Omega(z)=\sum_{\Omega_{i} \in \tau} R_{k i}^{(\tau)} S_{i},
$$

where, $R_{k i}^{(\tau)} \equiv \int_{\Omega_{i}} I_{k}\left(z-z_{c}\right) d \Omega(z)$. Here, we assumed $S(z)=S\left(z_{i}\right) \equiv S_{i}$ to be constant over $\Omega_{i}$. Integration yields,

$$
R_{k i}^{(\tau)}=\int_{-1}^{1} \int_{-1}^{1} \frac{\left(z-z_{c}\right)^{k}}{k !}\left|\frac{\partial z}{\partial s}\right|\left|\frac{\partial z}{\partial t}\right| d s d t=-i\left[\frac{\left(z_{1}-z_{c}\right)^{(k+2)}+\left(z_{4}-z_{c}\right)^{(k+2)}-\left(z_{2}-z_{c}\right)^{(k+2)}-\left(z_{3}-z_{c}\right)^{(k+2)}}{(k+2) !}\right] .
$$

\section{Appendix B.3. Stand-alone FMM for complex geometries}

For complex geometries, we split the original solution into inhomogeneous $\left(p^{i}\right)$ and homogeneous $\left(p^{h}\right)$ parts as described in Sec. 2 except that boundary conditions on $\Gamma_{c}$ (Eq. 3) are not imposed on $p^{i}$. First, a particular solution $p^{i}$ is computed in $\Omega_{c}$ through FMM intead of GMG.

$$
p^{i}\left(\boldsymbol{x}_{j}\right)=-\int_{\Omega_{c}} G\left(\boldsymbol{x}, \boldsymbol{x}_{j}\right) S(\boldsymbol{x}) d \Omega, \quad \boldsymbol{x}_{j} \in \Omega_{c} .
$$

Above integral is discretized and computed as described in the above subsections. Then, homogeneous solution is obtained through the BEM-FMM described earlier in Sec. 3-4. This approach avoids the otherwise necessary discretization of $\Omega$ into an unstructured (non-Cartesian) mesh while Cartesian mesh has computational advantages including efficient evaluation of $C_{j i}$ through analytical expressions Eq. (B.4)-(B.6) rather than expensive numerical quadratures. Note that analytical expressions for cells of other shapes such as triangles can be derived which is not the focus of this article.

\section{Acknowledgements}

The research reported in this publication was supported by funding from King Abdullah University of Science and Technology (KAUST).

\section{References}

Askham, T., Cerfon, A. J., 2017. An adaptive fast multipole accelerated Poisson solver for complex geometries. J. Comp. Phy. 344, 1-22.

Carrier, J., Greengard, L., Rokhlin, V., 1988. A fast adaptive multipole algorithm for particle simulations. SIAM J. Sci. Statist. Comput. 9, 669-686. Cheng, H., Greengard, L., Rokhlin, V., 1999. A fast adaptive multipole algorithm in three dimensions. J. Comp. Phy. 155, 468-498.

Coco, A., Russo, G., 2013. Finite-difference ghost-point multigrid methods on Cartesian grids for elliptic problems in arbitrary domains. J. Comp. Phy. 241, 464-501.

Coco, A., Russo, G., 2018. Second order finite-difference ghost-point multigrid methods for elliptic problems with disconsitnuous coefficients on an arbitrary interface. J. Comp. Phy. 361, 299-330.

Crockett, R. K., Colella, P., Graves, D. T., 2011. A Cartesian grid embedded boundary method for solving the Poisson and heat equations with discontinuous coefficients in three dimensions. J. Comp. Phy. 230 (7), 2451-2469.

Devendran, D., Graves, D. T., Johansen, H., Ligocki, T., 2017. A fourth-order Cartesian grid embedded boundary method for Poisson's equation. Comm. App. Math. Comp. Sci. 12 (1), 51-79.

Ethridge, F., Greengard, L., 2001. A new fast-multipole accelerated Poisson solver in two dimensions. SIAM J. Sci. Comput. 23 (3), $741-760$.

Gholami, A., Malhotra, D., Sundar, H., Biros, G., 2016. FFT, FMM, or multigrid? A comparative study of state-of-the-art Poisson solvers for uniform and nonuniform grids in the unit cube. SIAM J. Sci. Comput. 38 (3), C280-C306.

Guillet, T., Teyssier, R., 2011. A simple multigrid scheme for solving the Poisson equation with arbitrary domain boundaries. J. Comp. Phy. 230, $4756-4771$.

Gumerov, N. A., Duraiswami, R., 2004. Fast multipole methods for the Helmholtz equation in three dimensions. Elsevier Series in Electromagnetism. Elsevier Science, Amsterdam.

URL https://doi .org/10.1016/B978-0-08-044371-3.X5000-5 
Hosseinverdi, S., Fasel, H. F., 2018. An efficient, high-order method for solving Poisson equation for immersed boundaries: Combination of compact difference and multiscale multigrid methods. J. Comp. Phy. In Press, https://doi.org/10.1016/j.jcp.2018.08.006.

Kavouklis, C., Colella, P., 2019. Computation of volume potentials on structured grids via the method of local corrections. Comm. App. Math. Com. Sc. 14 (1), 1-32.

Langston, M. H., Greengard, L., Zorin, D., 2011. A free-space adaptive FMM-based PDE solver in three dimensions. Commun. Appli. Math. Comput. Sci. 6, 79-122.

Larsson, J., Lien, F. S., Yee, E., 2005. Conditional semicoarsening multigrid algorithm for the Poisson equation on anisotropic grids. J. Comp. Phy. 208, 368-383.

Liska, S., Colonius, T., 2014. A parallel fast multipole method for elliptic difference equations. J. Comp. Phy. $278,76-91$.

Liska, S., Colonius, T., 2016. A fast lattice Green's function method for solving viscous incompressible flows on unbounded domains. J. Comp. Phy. 316, 360-384.

Liska, S., Colonius, T., 2017. A fast immersed boundary method for external incompressible viscous flows using lattice green's functions. J. Comp. Phy. 331, 257-279.

Liu, Y. J., Nishimura, N., 2006. The fast multipole boundary element method for potential problems: A tutorial. Engineering Analysis with Boundary Elements 30 (5), 371-381.

Nishimura, N., 2002. Fast multipole accelerated boundary integral equation methods. Appl. Mech. Rev. 55, $299-324$.

Pozrikidis, C., 2002. A Practical Guide to Boundary Element Methods with the Software Library BEMLIB. CRC Press, Inc., Boca Raton, FL, USA.

Pozrikidis, C., 2008. Numerical Computation in Science and Engineering, 2nd Edition. Oxford University Press.

Rapaka, N. R., Samtaney, R., 2018. Non-normal stability of embedded boundary methods through pseudospectra. J. Comp. Phy. 373, 975-999.

Rapaka, N. R., Sarkar, S., 2016. An immersed boundary method for direct and large eddy simulation of stratified flows in complex geometry. J. Comp. Phy. 322, 511-534. 
Manuscript: An efficient Poisson solver for complex embedded boundary domains using the multi-grid and fast multipole methods by N. R. Rapaka and R. Samtaney

\section{Declaration of interests}

$\bigotimes$ The authors declare that they have no known competing financial interests or personal relationships that could have appeared to influence the work reported in this paper.

$\square$ The authors declare the following financial interests/personal relationships which may be considered as potential competing interests: 